\title{
Characteristic varieties of algebraic curves
}

\author{
A.LIBGOBER \\ Department of Mathematics, University of Illinois at Chicago, Chicago, Illinois, USA
}

September 2, 2018

\section{Introduction.}

A procedure for calculation of fundamental groups for the complements to algebraic curves in complex projective plane was found by Zariski and van Kampen ([Z],[vK]). Their methods yielded several important calculations and results on the fundamental groups of the complements (cf. for example [L2] for references). However, only limited information was obtained about their algebraic structure or what actually affects the complexity of these fundamental groups. This paper is a result of attempts to find alternative ways for calculating the fundamental groups of the complements or at least some invariants of these groups. The invariants of the fundamental groups, which we consider here, are certain subvarieties of complex tori $\mathbf{C}^{* r}$. They were called characteristic varieties in [L3]. These subvarieties are unions of translated subtori, as follows from recent work [Ar]. We calculate these subtori in terms of local type of singularities and dimensions of linear systems which we attach to the configuration of singularities of the curve.

These characteristic varieties can be defined as follows. Let $\mathcal{C}=\cup_{1 \leq i \leq r} C_{i}$ be an algebraic curve in $\mathbf{C}^{2}$ and $\pi_{1}=\pi_{1}\left(\mathbf{C}^{2}-\mathcal{C}\right)$ be the fundamental group of its complement. Then $\pi_{1} / \pi_{1}^{\prime}$ is isomorphic to $\mathbf{Z}^{r}$ and acts on $\pi_{1}^{\prime} / \pi_{1}^{\prime \prime}$ by conjugation. This makes $\pi_{1}^{\prime} / \pi_{1}^{\prime \prime}$ into a module over the group ring of $\pi_{1} / \pi_{1}^{\prime}$. The latter is just the ring of Laurent polynomials $\mathbf{Z}\left[t_{1}, t_{1}^{-1}, \ldots, t_{r}, t_{r}^{-1}\right]$. After tensoring with $\mathbf{C}$, we obtain a $\mathbf{C}\left[\pi_{1} / \pi_{1}^{\prime}\right]$-module $\pi_{1}^{\prime} / \pi_{1}^{\prime \prime} \otimes \mathbf{C}$. The support of 
its $i-t h$ exterior power is a subvariety of the torus $\operatorname{Spec} \mathbf{Z}\left[t_{1}, t_{1}^{-1}, \ldots, t_{r}, t_{r}^{-1}\right]$ called the i-th characteristic variety of $\mathcal{C}: \operatorname{Char}_{i}(\mathcal{C})($ cf. [L3]).

This invariant of the fundamental group can be used to calculate the homology of abelian covers of $\mathbf{C}^{2}-\mathcal{C}$ (cf. [L3]) and covers of $\mathbf{P}^{2}$ branched over the projective closure of $\mathcal{C}$ ( $\mathrm{cf}$. [Sa]). The above construction of characteristic varieties can be, of course, carried out for any topological space with $H_{1}=\mathbf{Z}^{r}$ and several known results can be recasted using them. For example, the modules $\pi_{1}^{\prime} / \pi_{1}^{\prime \prime}$ were widely studied in the context of the complements to links in spheres (cf. e.g. [Hil] and references there; for the case of algebraic links cf. [Sab]). In this case, $\mathrm{Char}_{1}$ is the set of zeros of the multi-variable Alexander polynomial. We shall see, however, that the characteristic varieties of algebraic curves rarely have codimension equal to one and hence cannot be described using single polynomial. The varieties $\operatorname{Char}_{i}(\mathcal{C})$ coincide with the cohomology support loci for local systems of rank 1 considered in [Ar]. The homology of the Milnor fiber of the function obtained by homogenizing a defining equation of $\mathcal{C}$ (i.e. the Milnor fiber of the cone over the projective closure of $\mathcal{C}$ ) can be found from the characteristic varieties of the latter. These Milnor fibers earlier were considered in the case when $\mathcal{C}$ is an arrangement i.e. when all components of $\mathcal{C}$ are lines (cf. [CS]).

For an irreducible $\mathcal{C}$ the characteristic varieties are subsets of $\mathbf{C}^{*}$ i.e. collections of complex numbers. Those are the roots of the Alexander polynomial of $\mathcal{C}$ (cf. [L1]) and the results of this paper are equivalent to the results of [L2].

Our calculation of $\mathrm{Char}_{i}$ based on the following observations. Firstly, by Arapura's theorem the characteristic varieties are translated tori and hence can be described by simple discrete data. Secondly, Sakuma's formula (cf. (1.3.2.2)), relating the homology of abelian branched over $\mathcal{C}$ cover to the characteristic varieties of $\mathcal{C}$, can be used to calculate such data for essential components (cf. 1.4.3)) of characteristic varieties completely from the information about the homology of all abelian covers with the branching locus $\mathcal{C}$. Thirdly, these abelian covers can be realized as complete intersections and one can use the theory of adjoints (cf. (1.5)) to calculate the homology of these covers (generalizing the calculations in the case of hypersurfaces cf. [Z], [L2], [L5]; complete intersections were used by Ishida (cf. [I], also $[\mathrm{Zu}])$ in similar context for calculations in the case of abelian covers of $\mathbf{P}^{2}$ branched along some arrangements). We associate with each singular point a collection of polytopes in the unit cube $\mathcal{U}$ in $\mathbf{R}^{r}$ union of which is $\mathcal{U}$ and 
call them the local polytopes of quasiadjunction (cf. sect. 2.4.1). Moreover, every such polytope defines the ideal in the local ring of the singular point. The collection of local polytopes defines new partition of the unit cube which is a refinement of partitions corresponding to all singular points of $\mathcal{C}$ and reflecting the global information about singularities of $\mathcal{C}$ (cf. sect. 2.6). We call the polytopes of this partition the global polytopes of quasiadjunction. In the set of faces of global polytopes of quasiadjnction we single out a subset of contributing ones. To each contributing face $\delta$ corresponds the linear system $H^{0}\left(\mathbf{P}^{2}, \mathcal{J}_{\delta}(\operatorname{deg} \mathcal{C}-3-l(\delta))\right.$ where the ideal sheaf $\mathcal{J}_{\delta} \subset \mathcal{O}_{\mathbf{P}^{2}}$ and the integer $l(\delta)$ are determined by the face $\delta$. The components of $\operatorname{Char}_{i} \mathcal{C}$ correspond to contributing faces of global polytopes of quasiadjunction for which $\operatorname{dim} H^{1}\left(\mathbf{P}^{2}, \mathcal{J}_{\delta}(\operatorname{deg} \mathcal{C}-3-l(\delta))\right)=i$. The main result of the paper is the theorem 3.1 where the equations for translated tori are given explicitly in terms of corresponding faces of polytopes of quasiadjunction (cf. sect. 3).

The procedure for calculating the characteristic varieties, though involving possibly large calculations, is entirely algorithmic. For example, suppose that the curve $\mathcal{C}$ is an arrangement of $r$ lines. (cf. sect. 3.3 for several examples of explicite calculations for such arrangements including Ceva's and Hesse's arrangements). Then any component of the characteristic variety $\operatorname{Char}_{i}(\mathcal{C})$, having positive dimension either belongs to a component of characteristic variety of a sub-arrangement (i.e. is inessential and can be found by applying this algorithm to a sub-arrangement) or is a connected component of a subgroup corresponding to a collection $\mathcal{S}$ of vertices in arrangement having multiplicity greater than 2 (more detailed calculation allows to pick the component as well, cf. th.3.1). A collection $\mathcal{S}$ yields a component of characteristic variety if it satisfies the following conditions.

a) Certain system of linear homogeneous equations attached to $\mathcal{S}$ has a non zero solution. This system constructed as follows: The unknowns $x_{i}$ are in one to one correspondence with $r(\mathcal{S})$ lines of arrangement containing points from $\mathcal{S}$. The equations are in one to one correspondence with the elements of $\mathcal{S}$. Left hand side of each equation is the sum of variables corresponding to the lines through a point of $\mathcal{S}$ and the right hand side is a positive integer (not exceeding the number of lines in the arramgements).

b) The set of solutions of the system from a) belongs to a hyperplane $\sum_{i=1}^{r(\mathcal{S})} x_{i}=l(\mathcal{S})$.

c) Let $\mathcal{I}_{\mathcal{S}}$ be the ideal sheaf with $\operatorname{Supp} \mathcal{O}_{\mathbf{P}^{2}} / \mathcal{I}_{\mathcal{S}}=\mathcal{S}$ which stalk at $P \in \mathcal{S}$ is 
$\mathcal{M}_{P}^{m-2-\rho(P)}$ where $\mathcal{M}_{P}$ is the maximal ideal of $P, m$ is the number of lines in $\mathcal{S}$ containing $P$ and $\rho(P)$ is the right hand side of the equaition in the system from a) corresponding to $P$. Then $\operatorname{dim} H^{1}\left(\mathbf{P}^{2}, \mathcal{I}_{\mathcal{S}}(r-3-l(\mathcal{S}))\right)=i \neq 0$.

Moreover if a)-c) are satisfied then the corresponding to $\mathcal{S}$ subgroup is the set of solutions of $\exp \left(L_{P}\right)=1$ where $L_{P}=\rho(P)$ are the equations of the system mentioned in a). Selection of particular connected component follows from more technical description in theorem 3.1

This algorithm yields complete description of characteristic tori of positive dimension (and essential torsion points). An interesting problem which left unanswered here is the realization problem: which tori (or collections of tori) can appear as characteristic tori of an algebraic curve with fixed degrees of components and given local type of singularities. Some information in this direction however is provided in section 4. Finally in the case of line arrangements (or equivalently the case of fundamental groups of the complements to arbitrary arrangements) the characteristic varieties give new sufficient conditions (resonance conditions) for Aomoto complex of an arrangement to be quasi-isomorphic to the corresponding twisted DeRham complex (in many situations less restrictive than previously used (cf. [ESV]). We describe in a new way the space of "resonant" Aomoto complexes on given arrangement i.e. those with the cohomology different from the cohomology of generic Aomoto complexes (th. 5.4.1; this space was considered in $[\mathrm{F}]$ ). Vice versa, this relation between the space of resonant Aomoto complexes and characteristic varieties shows that components of characteristic varieties which are subgroups of the group of characters are combinatorial invariants of arrangements. Moreover, Aomoto complexes provide another algorithm for calculating these components of characteristic varieties of the fundamental groups of the complements to arrangements.

The announcement of these results is presented in [L6]. This work was supported by NSF grants DMS-9803623, DMS-9872025 and Mittag Leffler Institute. I am also grateful to S.Yuzvinsky for very interesting correspondence regarding the material in section 5 and to J.Cogolludo for useful discussions of the manuscript.

\section{Preliminaries}




\subsection{Setting.}

Let $\overline{\mathcal{C}}=\cup \bar{C}_{i}(i=1, \ldots r)$ be a reduced algebraic curve in $\mathbf{P}^{2}$ where $\bar{C}_{i}(i=$ $1, \ldots r)$ are the irreducible components of $\overline{\mathcal{C}}$. We shall denote by $d_{i}$ the degree of the component $\bar{C}_{i}$. Let $L_{\infty}$ be a line in $\mathbf{P}^{2}$ which we shall view as the line at infinity. We shall be concerned with the fundamental groups of the complements to $\overline{\mathcal{C}}$ in $\mathbf{P}^{2}$ and in $\mathbf{C}^{2}=\mathbf{P}^{2}-L_{\infty}$. Let $\mathcal{C}=\cup C_{i}$ be the affine portion of $\overline{\mathcal{C}}$. The homology of these complements are the following (cf. [L1]):

$$
H_{1}\left(\mathbf{C}^{2}-\mathcal{C}, \mathbf{Z}\right)=\mathbf{Z}^{r}, \quad H_{1}\left(\mathbf{P}^{2}-\overline{\mathcal{C}}, \mathbf{Z}\right)=\mathbf{Z}^{r} /\left(d_{1}, \ldots, d_{r}\right)
$$

Generators of these homology groups are represented by the classes of the loops $\gamma_{i}$, each of which is the boundary of a small 2-disk intersecting $C_{i}$ (resp. $\bar{C}_{i}$ ) transversally at a non singular point.

For the fundamental groups we have the exact sequence:

$$
\pi_{1}\left(\mathbf{C}^{2}-\mathcal{C}\right) \rightarrow \pi_{1}\left(\mathbf{P}^{2}-\overline{\mathcal{C}}\right) \rightarrow 1
$$

If the line $L_{\infty}$ is transversal to $\overline{\mathcal{C}}$, then the kernel of the surjection (1.1.2) is isomorphic to $\mathbf{Z}$ and belongs to the center of $\pi_{1}\left(\mathbf{C}^{2}-\mathcal{C}\right)$ (cf. [L4]). In general, the fundamental group of the affine portion of the complement to $\overline{\mathcal{C}}$ in $\mathbf{P}^{2}$ depends on position of $L_{\infty}$ relative to $\overline{\mathcal{C}}$. Throughout the paper we assume that $L_{\infty}$ is transversal to $\overline{\mathcal{C}}$.

\subsection{Characteristic varieties of algebraic curves.}

\section{2 .1}

Let $R$ be a commutative Noetherian ring and $M$ be a finitely generated $R$ module. Let $\Phi: R^{m} \rightarrow R^{n}$ be such that $M=$ Coker $\Phi$. Recall that the $k$-th Fitting ideal of $M$ is the ideal generated by $(n-k+1) \times(n-k+1)$ minors of the matrix of $\Phi$ (clearly depending only on $M$ rather than on $\Phi)$. The $k$-th characteristic variety $M$ is the reduced sub-scheme of $\operatorname{Spec} R$ defined by $F_{k}(M)$.

If $R=\mathbf{C}[H]$ where $H$ is a free abelian group then $R$ can be identified with the ring of Laurent polynomials and $\operatorname{Spec} R$ is a complex torus. In particular each $k$-th characteristic variety of an $R$-module is a subvariety $V_{k}(M)$ of $\left(\mathbf{C}^{*}\right)^{\mathrm{rk} H}$. 
If $A n n \wedge^{k} M \subset R$ is the annihilator of the $k$-th exterior power of $M$ then (cf. [BE], Cor.1.3): $\left(A n n \wedge^{k} M\right)^{t} \subseteq F_{k}(M) \subseteq A n n \wedge^{k} M$ for some integer $t$. In particular, if $\operatorname{Supp}(M) \subset \operatorname{Spec}(R)$ is the set of prime ideals in $R$ containing $\operatorname{Ann}(M)$ (alternatively $\{\wp \in \operatorname{Spec} R \mid M \otimes R / \wp R \neq 0\}$, cf. [Se] p.3), then $\operatorname{Supp}\left(\wedge^{k} M\right)=\operatorname{Supp}\left(R / F_{k}(M)\right)$ is the $k$-th characteristic variety of $M$.

Note the following:

Lemma 1.2.1 Let $0 \rightarrow M^{\prime} \rightarrow M \rightarrow M^{\prime \prime} \rightarrow 0$. Then $V_{1}(M)=V_{1}\left(M^{\prime}\right) \cup$ $V_{1}\left(M^{\prime \prime}\right)$ and for $k \geq 2: V_{k}\left(M^{\prime \prime}\right) \subset V_{k}(M) \subset V_{k}\left(M^{\prime \prime}\right) \cup V_{k-1}\left(M^{\prime \prime}\right) \cap V_{1}\left(M^{\prime}\right)$.

The first equality is Prop. 4(a) in [Se]. The second follows from the first and the exact sequence: $\Lambda^{k-1} M^{\prime \prime} \otimes M^{\prime} \rightarrow \Lambda^{k}(M) \rightarrow \Lambda^{k}\left(M^{\prime \prime}\right) \rightarrow 0$, since $\operatorname{Supp}(A \otimes B)=\operatorname{Supp}(A) \cap \operatorname{Supp}(B)$ for any $R$-modules of finite type (cf. [Se], Prop. 4(c)).

\subsection{2}

Let $G$ be a finitely generated, finitely presented group such that $H_{1}(G, \mathbf{Z})=$ $G / G^{\prime}=\mathbf{Z}^{r}$ (for example $G=\pi_{1}\left(\mathbf{C}^{2}-\mathcal{C}\right.$ ) where $\mathcal{C}=\cup C_{i}$ is a plane curve as in 1.1; another class of examples which was studied in detail is given by link groups, cf. [Hil]). Then $G^{\prime} / G^{\prime \prime} \otimes \mathbf{C}$ can be viewed as $H_{1}(\tilde{X}, \mathbf{C})$ where $X$ is a topological space with $\pi_{1}(X)=G$ and $\tilde{X}$ is the universal abelian cover of $X$. The group $G / G^{\prime}=H_{1}(X, \mathbf{Z})$ acts as the group of deck transformations on $\tilde{X}$ and hence $G^{\prime} / G^{\prime \prime} \otimes \mathbf{C}$ has a structure of a $\mathbf{C}\left[G / G^{\prime}\right]$-module. We shall denote the i-th characteristic variety of this module as $V_{i}(G)$ (or $V_{i}(\mathcal{C})$ if $G=\pi_{1}\left(\mathbf{C}^{2}-\mathcal{C}\right)$ ) and call it the i-th characteristic variety of $G$ (resp. $\left.\mathcal{C}\right)$. The depth of a component $V$ is the integer $i=\max \left\{j \mid V \subset V_{j}(G)\right\}$. We shall see below that if a component has depth $i$ and dimension $\varrho>0$ and contains identity, then $i=\varrho-1$, cf. footnote in 1.4.2.

1.2.2.1.

If $G=F_{r}$ is a free group on $r$-generators then $G^{\prime} / G^{\prime \prime}=H_{1}\left(\widetilde{\bigvee_{r} S^{1}}, \mathbf{Z}\right)$, where $\widetilde{\mathrm{V}_{r}} S^{1}$ is the universal abelian cover of the wedge of $r$ circles. It fits into the exact sequence:

$$
0 \rightarrow H_{1}\left(\widetilde{\bigvee_{r}} S^{1}, \mathbf{C}\right) \rightarrow \mathbf{C}\left[\mathbf{Z}^{r}\right]^{r} \rightarrow I \rightarrow 0
$$


with $I$ denoting the augmentation ideal of the group ring of $\mathbf{Z}^{r}$. (As an universal abelian cover of $\widetilde{\bigvee_{r}} S^{1}$ one can take the subset of $\mathbf{R}^{r}$ of points having at least $r-1$ integer coordinates with the action of $\mathbf{Z}^{r}$ given by translations; unit vectors of the standard basis provide identification of 1-chains with $\mathbf{C}\left[\mathbf{Z}^{r}\right]^{r}$ while the module of 0 -chains is identified with $\mathbf{C}\left[\mathbf{Z}^{r}\right]$ ). This shows that $H_{1}\left(\widetilde{\mathrm{V}_{r} S^{1}}, \mathbf{C}\right)$ is cokernel of the map $\Lambda^{\left(\begin{array}{c}r \\ 3\end{array}\right)} \mathbf{C}\left[\mathbf{Z}^{r}\right]^{r} \rightarrow \Lambda^{\left(\begin{array}{c}r \\ 2\end{array}\right)} \mathbf{C}\left[\mathbf{Z}^{r}\right]^{r}$ in the Koszul resolution corresponding to the $\left(x_{1}-1\right), \ldots,\left(x_{r}-1\right)$. This implies that $V_{i}\left(F_{r}\right)=\mathbf{C}^{* r}$ for $0<i \leq r-1$ and $V_{i}\left(F_{r}\right)=(1, \ldots, 1)$ for $r \leq i \leq\left(\begin{array}{l}r \\ 2\end{array}\right)$ (cf. also (1.4.1) below).

If $G$ is the fundamental group of a link in a 3-sphere $S^{3}$ with $r$ components, then the first determinantal ideal is generated by $\left(t_{1}-1\right), \ldots,\left(t_{r}-1\right)$ and certain principal ideal. A generator $\Delta\left(t_{1}, \ldots, t_{r}\right)$ of the latter is called the Alexander polynomial. Alexander polynomial satisfies $\Delta(1, \ldots, 1)=0$ and hence $V_{1}(G)$ is the hypersurface $\Delta\left(t_{1}, \ldots, t_{r}\right)=0$. Extensive calculations of the Alexander polynomials of links can be found in [SW]. In particular, if $G$ is the fundamental group of the complement to the Hopf link in $S^{3}$ with $r$ components then $V_{1}(G)$ is the set of zeros of $t_{1} \cdot t_{2} \cdots t_{r}-1$. Moreover $V_{1}(G)=\ldots=V_{r-1}(G)$ (cf. [L4],p.165). From a presentation of $G$ using Fox calculus one can calculate a presentation of $\pi_{1}^{\prime} / \pi_{1}^{\prime \prime} \otimes \mathbf{C}$ as a $\mathbf{C}([H])$-module and hence the characteristic varieties of $\pi_{1}$ (cf. [Hi], [CS] for examples of such calculations).

\section{2 .3}

Let $T\left(L_{\infty}\right)$ be a small tubular neighborhood of $L_{\infty}$ in $\mathbf{P}^{2}$. If $\partial T\left(L_{\infty}\right)$ is its boundary then $\pi_{1}\left(\partial T\left(L_{\infty}\right)-\partial T\left(L_{\infty}\right) \cap \mathcal{C}\right) \rightarrow \pi_{1}\left(\mathbf{P}^{2}-L_{\infty} \cup \overline{\mathcal{C}}\right)$ is a surjection. Lemma 1.2.1 implies that the characteristic variety of $\mathcal{C}$ is a subset of the torus $t_{1}^{d_{1}} \cdots t_{r}^{d_{r}}=1$ since $\mathcal{C} \cap \partial T\left(L_{\infty}\right) \subset \partial T\left(L_{\infty}\right)=S^{3}$ is the Hopf link with $d_{1}+\ldots+d_{r}$ components with $d_{i}$ components of the link belonging to $C_{i}$ and hence corresponding to $t_{i}$ for each $1 \leq i \leq r$ (cf. [L3]). In fact the characteristic varieties of an affine curve can be determined from the projectivization as follows.

It is a corollary of (1.1.1) that $\mathbf{T}_{a}=\operatorname{Spec} \mathbf{C}\left[H_{1}\left(\mathbf{C}^{2}-\mathcal{C}\right)\right]$ is the torus of dimension $r$ and that $\mathbf{T}_{p}=\operatorname{Spec} \mathbf{C}\left[H_{1}\left(\mathbf{P}^{2}-\overline{\mathcal{C}}\right)\right]$ is the sub-scheme of zeros of $t_{1}^{d_{1}} \cdots t_{r}^{d_{r}}-1$ in $\mathbf{T}_{a}$. We denote by $E: \mathbf{T}_{p} \rightarrow \mathbf{T}_{a}$ the corresponding embedding. On the other hand, the construction of (1.2.1) and (1.2.2) yields subvarieties 
$V_{i}(\overline{\mathcal{C}})_{p}$ in $\mathbf{T}_{p}$

Proposition 1.2.3 The characteristic variety of projective and affine curves satisfy:

$$
V_{i}(\mathcal{C})=E\left(V_{i}(\overline{\mathcal{C}})_{p}\right)
$$

Proof. It follows from the isomorphism:

$$
\pi_{1}^{\prime}\left(\mathbf{P}^{2}-\overline{\mathcal{C}}\right) / \pi_{1}^{\prime \prime}\left(\mathbf{P}^{2}-\overline{\mathcal{C}}\right)=\pi_{1}^{\prime}\left(\mathbf{C}^{2}-\mathcal{C}\right) / \pi_{1}^{\prime \prime}\left(\mathbf{C}^{2}-\mathcal{C}\right)
$$

equivariant with respect to the action of $H_{1}\left(\mathbf{C}^{2}-\mathcal{C}\right)$. This isomorphism is a consequence of (1.1.2) because in the latter the left map induces isomorphism on commutators. Indeed, the kernel of surjection (1.1.2) is isomorphic to $\mathbf{Z}$ (cf. (1.1)) and does not intersect $\pi_{1}^{\prime}\left(\mathbf{C}^{2}-\mathcal{C}\right)$ because it injects into $H_{1}\left(\mathbf{C}^{2}-\mathcal{C}\right)$ (cf. also [L4]).

\subsection{Abelian covers}

\section{3 .1}

Let $m_{1}, \ldots, m_{r}$ be positive integers and $h_{m_{1}, . ., m_{r}}: H_{1}\left(\mathbf{C}^{2}-\mathcal{C}, \mathbf{Z}\right) \rightarrow \mathbf{Z} / m_{1} \mathbf{Z} \oplus$ $\ldots \oplus \mathbf{Z} / m_{r} \mathbf{Z}$ be the surjection $\gamma_{i} \rightarrow \gamma_{i} \bmod m_{i}$. The kernel of the homomorphism $\pi_{1}\left(\mathbf{C}^{2}-\mathcal{C}\right) \rightarrow \mathbf{Z} / m_{1} \mathbf{Z} \oplus \ldots \oplus \mathbf{Z} / m_{r} \mathbf{Z}$, which is the composition of the abelianization $a b: \pi_{1}\left(\mathbf{C}^{2}-\mathcal{C}\right) \rightarrow H_{1}\left(\mathbf{C}^{2}-\mathcal{C}\right)$ and $h_{m_{1}, \ldots, m_{r}}$, defines an unbranched cover of $\mathbf{C}^{2}-\mathcal{C}$. We shall denote it as $\left(\mathbf{C}^{2}-\widetilde{\mathcal{C}}\right)_{m_{1}, \ldots, m_{r}}$. This is a quasi-projective algebraic variety defining a birational class of projective surfaces $\overline{\left(\mathbf{C}^{2}-\widetilde{\mathcal{C})_{m_{1}, \ldots, m_{r}}}\right.}$. Birational invariants of surfaces in this class (in particular the first Betti number of a non singular model) depend only on $\mathcal{C}$ and the homomorphism $h_{m_{1}, \ldots, m_{r}}$.

If $h_{m_{1}, \ldots, m_{r}}\left(d_{1} \gamma_{1}+\ldots d_{r} \gamma_{r}\right)=0$, then the corresponding branched covering of $\mathbf{C}^{2}$ is a restriction of the covering of $\mathbf{P}^{2}$ unbranched over the line at infinity. It can be easily checked that the first Betti numbers of those two branched coverings are the same, since we assume (cf. (1.1)) that the line at infinity is transversal to $\mathcal{C}$ (cf. [L1]).

A model (singular, in general) for a surface birational to $\overline{\left(\mathbf{C}^{2}-\widetilde{\mathcal{C}}\right)_{m_{1}, \ldots, m_{r}}}$ can be constructed as follows. Let $f_{i}(u, x, y)=0$ be an equation of the 
component $C_{i}(i=1, \ldots, r)$. Let $V_{m_{1}, \ldots, m_{r}}$ be a complete intersection on $\mathbf{P}^{r+2}$ (coordinates of which we shall denote $z_{1}, . ., z_{r}, u, x, y$ ) given by the equations

$$
z_{1}^{m_{1}}=u^{m_{1}-d_{1}} f_{1}(u, x, y), \ldots, z_{r}^{m_{r}}=u^{m_{r}-d_{r}} f_{r}(u, x, y)
$$

Projection from the subspace given by $u=x=y=0$ onto the plane $z_{1}=\ldots=z_{r}=0$ (i.e. $\left(z_{1}, . ., z_{r}, u, x, y\right) \rightarrow(u, x, y)$ ), when restricted on the preimage in $V_{m_{1}, \ldots, m_{r}}$ of $\mathbf{C}^{2}-\mathcal{C}$, is unbranched cover of $\mathbf{C}^{2}-\mathcal{C}$ corresponding to $\operatorname{Ker}\left(h_{m_{1}, \ldots, m_{r}} \circ a b\right)$.

\section{3 .2}

The first Betti number of unbranched cover $\left(\mathbf{C}^{2}-\widetilde{\mathcal{C})_{m_{1}, \ldots, m_{r}}}\right.$ can be found in terms of the characteristic varieties of $\mathcal{C}$ as follows (cf. [L3]). For $P \in \mathbf{C}^{* r}$ let $f(P, \mathcal{C})=\left\{\max \quad i \mid P \in V_{i}(\mathcal{C})\right\}$. Then

$$
b_{1}\left(\left(\mathbf{C}^{2}-\widetilde{\mathcal{C})_{m_{1}, \ldots, m_{r}}}\right)=r+\Sigma_{\omega_{i}^{m_{i}}=1,\left(\omega_{m_{1}}, \ldots, \omega_{m_{r}}\right) \neq(1, \ldots, 1)} f\left(\left(\omega_{m_{1}}, . ., \omega_{m_{r}}\right), \mathcal{C}\right)\right.
$$

The first Betti number of a resolution of branched cover of $\mathbf{P}^{2}$ (i.e. of $\left.V_{m_{1}, \ldots, m_{r}}\right)$ can be calculated using the characteristic varieties of curves formed by components of $\mathcal{C}$ (cf. [Sa]). Let $\widetilde{V}_{m_{1}, . ., m_{r}}$ be such a resolution. For a torsion point of $\omega=\left(\omega_{1}, \ldots, \omega_{r}\right), \omega_{i}^{m_{i}}=1$ in the torus $\mathbf{C}^{* r}$ let $\mathcal{C}_{\omega}=\cup_{i \mid \omega_{i} \neq 1} C_{i}$. Then the first Betti number of $\widetilde{V}_{m_{1}, \ldots, m_{r}}$ equals:

$$
\Sigma_{\omega} \max \left\{i \mid \omega \in \operatorname{Char}_{i}\left(\mathcal{C}_{\omega}\right)\right\}
$$

More precisely, if $\chi_{\omega}$ is the character of $\pi_{1}\left(\mathbf{C}^{2}-\mathcal{C}\right)$ such that $\chi_{\omega}\left(\gamma_{i}\right)=\omega_{i}$ and for a character $\chi$ of the Galois group $\operatorname{Gal}\left(\widetilde{V}_{m_{1}, \ldots, m_{r}} / \mathbf{P}^{2}\right)$ we put:

$H_{1, \chi}\left(\widetilde{V}_{m_{1}, \ldots, m_{r}}\right)=\left\{x \in H_{1}\left(\widetilde{V}_{m_{1}, \ldots, m_{r}}\right) \mid g(x)=\chi(g) \cdot x, \forall g \in \operatorname{Gal}\left(\widetilde{V}_{m_{1}, \ldots, m_{r}} / \mathbf{P}^{2}\right)\right\}$

then

$$
\operatorname{dim} H_{1, \chi_{\omega}}=\max \left\{i \mid \omega \in \operatorname{Char}_{i}\left(C_{\omega}\right)\right\}
$$




\subsubsection{A bound on the growth of Betti number.}

Proposition 1.3.3 Let $b_{1}(\overline{\mathcal{C}}, n)$ (resp. $b_{1}(\mathcal{C}, n)$ ) be the first Betti number of the cover of $\mathbf{P}^{2}$ (resp. $\mathbf{C}^{2}-\mathcal{C}$ ) branched over $L_{\infty} \cup \overline{\mathcal{C}}$ (resp. unbranched) and corresponding to the surjection $h_{n, \ldots, n}: \pi_{1}\left(\mathbf{P}^{2}-L_{\infty} \cup \overline{\mathcal{C}}\right) \rightarrow(\mathbf{Z} / n \mathbf{Z})^{r}$ (given by evaluation modulo $n$ of the linking numbers of loops with the components of $\mathcal{C}$ modulo $n)$. Then $b_{1}(\overline{\mathcal{C}}, n) \leq \bar{C}_{1} \cdot n^{r-1}$. (resp. $\left.b_{1}(\mathcal{C}, n) \leq C_{1} \cdot n^{r-1}\right)$ for some constants $C_{1}, \bar{C}_{1}$ independent of $n$.

Proof. This follows from the Sakuma's formula (1.3.2.2) (resp. (1.3.2.1)) and the obvious remark that the number of $n$-torsion points on a torus of dimension $l$ grows as $n^{l}$ since $\operatorname{dim}\left(\operatorname{Char}_{i}\left(\pi_{1}\left(\mathbf{C}^{2}-\mathcal{C}\right)^{\prime} / \pi_{1}\left(\mathbf{C}^{2}-\mathcal{C}\right)^{\prime \prime}\right) \leq r-1\right.$ by 1.2 .3 .

\subsubsection{Characteristic varieties and the homology of Milnor fibers.}

The polynomial $f_{1}(u, x, y) \cdots f_{r}(u, x, y)$ (which set of zeros in $\mathbf{P}^{2}$ is $\overline{\mathcal{C}}$ ) defines a cone in $\mathbf{C}^{3}$ having a non isolated singularity, provided $\mathcal{C}$ is singular. The Milnor fiber $M_{c}$ of this singularity (cf. [CS] in the case when $\operatorname{deg} f_{i}=1, \forall i$ ) is diffeomorphic to an affine hypersurface given by the equation: $f_{1} \cdots f_{r}=c, c \neq 0$. Quotient of the latter by the action of the cyclic group $\mathbf{Z} / d \mathbf{Z}\left(d=\Sigma_{i} d_{i}, d_{i}=\right.$ $\left.\operatorname{deg} f_{i}\right)$ acting via $(u, x, y) \rightarrow\left(\omega_{d} u, \omega_{d} x, \omega_{d} y\right), \omega_{d}^{d}=1$ is $\mathbf{P}^{2}-\overline{\mathcal{C}}$. In other words, the Milnor fiber is the cyclic cover $p: M_{c} \rightarrow \mathbf{P}^{2}-\overline{\mathcal{C}}$ corresponding to the homomorphism sending $\gamma_{i} \rightarrow 1 \bmod d$. The exact sequence of the pair $\left(M_{c}, p^{-1}\left(\mathbf{P}^{2}-\overline{\mathcal{C}} \cup L_{\infty}\right)\right)$ shows that $r k H_{1}\left(M_{c}\right)=r k H_{1}\left(p^{-1}\left(\mathbf{P}^{2}-\overline{\mathcal{C}} \cup L_{\infty}\right)-1\right.$, since we assume that $\overline{\mathcal{C}}$ is transversal to $L_{\infty}$ (cf. [L1]). Hence it follows from (1.3.2.1) that

$$
r k H_{1}\left(M_{c}\right)=r-1+\Sigma_{i=1}^{d-1} f\left(\left(\omega_{d}^{i}, . ., \omega_{d}^{i}\right), \mathcal{C}\right)
$$

\subsection{Characteristic varieties and support loci for rank one local systems}




\section{4 .1}

Let again $G$ be a group such that $G / G^{\prime}=\mathbf{Z}^{r}$. If $X$ is a topological space with $\pi_{1}(X)=G$ then the local systems of rank one on $X$ correspond to the points $\operatorname{Hom}\left(G, \mathbf{C}^{*}\right)$ (cf. [St]). The latter has a natural identification with $H^{1}\left(X, \mathbf{C}^{*}\right)$. Each $\gamma_{i}$, corresponding to a component $C_{i}$ of $\mathcal{C}$ (cf. 1.1), defines the homomorphism $t_{i}: \operatorname{Hom}\left(G, \mathbf{C}^{*}\right) \rightarrow \mathbf{C}^{*}$ given by $t_{i}(\chi)=\chi\left(\gamma_{i}\right), \chi \in$ $\operatorname{Hom}\left(G, \mathbf{C}^{*}\right)$. Therefore $t_{i}$ 's provide an identification of $\operatorname{Hom}\left(G, \mathbf{C}^{*}\right)$ with $\mathrm{C}^{* r}$.

The homology groups $H_{i}(X, \rho)$ of $X$ with coefficients in a local system corresponding to a homomorphism $\rho: \pi_{1}(X) \rightarrow H_{1}(X, \mathbf{Z}) \rightarrow \mathbf{C}^{*}$ are the homology of the complex $C_{i}(\tilde{X}) \otimes_{H_{1}(X, \mathbf{Z})} \mathbf{C}$ where $\mathbf{C}$ is equipped with the structure of $\mathbf{Z}\left[H_{1}(X, \mathbf{Z})\right]$-module using $\rho$. If $\rho \neq 1$ then

$$
H_{1}(\tilde{X}, \mathbf{C}) \otimes_{\mathbf{C}\left[H_{1}(X, \mathbf{Z})\right]} \mathbf{C}=H_{1}(X, \rho)
$$

This follows, for example, from the exact sequence of the low degree terms in the spectral sequence corresponding to the action of $H_{1}(X, \mathbf{Z})$ on the universal abelian cover $\tilde{X}: H_{p}\left(H_{1}(X, \mathbf{Z}), H_{q}(\tilde{X})_{\rho}\right) \Rightarrow H_{p+q}(X, \rho)$ (here $H_{q}(\tilde{X})_{\rho}$ denotes the homology of the complex $C_{i}(\tilde{X}) \otimes_{\mathbf{Z}} \mathbf{C}$ with the action of $H_{1}(X, \mathbf{Z})$ given by $g(e \otimes \alpha)=g \cdot e \otimes \rho\left(g^{-1}\right) \alpha, g \in H_{1}(X, \mathbf{Z}), e \in C_{i}(\tilde{X}), \alpha \in \mathbf{C}$ i.e. the usual homology $H_{q}(\tilde{X}, \mathbf{C})$ with the action of $H_{1}(X, \mathbf{Z})$ changed by the character $\rho$ (cf. [CE],ch. XVI,th. 8.4). This exact sequence is:

$H_{2}(X, \rho) \rightarrow H_{2}\left(H_{1}(X, \mathbf{Z}), \rho\right) \rightarrow\left(H_{1}(\tilde{X})_{\rho}\right)_{H_{1}(X, \mathbf{Z})} \rightarrow H_{1}(X, \rho) \rightarrow H_{1}\left(H_{1}(X, \mathbf{Z}), \rho\right) \rightarrow 0$

(cf. [CE], ch XVI, (4a)). Since for $\rho \neq 1$ we have $H_{i}\left(H_{1}(X, \mathbf{Z}), \rho\right)=0$, we obtain (1.4.1.1). For $\rho=1$, an argument similar to [L3], sect. 1 yields that $\operatorname{dim}_{1}(\tilde{X}, \mathbf{C}) \otimes_{\mathbf{C}\left[H_{1}(X, \mathbf{Z})\right]} \mathbf{C}$ is the dimension of the kernel of the map $\cup_{X}: \Lambda^{2} H^{1}(X, \mathbf{C}) \rightarrow H^{2}(X, \mathbf{C})$ given by the cup product. From the definition of Fitting ideals (cf. 1.2.1) it follows that for $\rho \neq 1$ one has:

$$
V_{i}(X)=\left\{\rho \in \operatorname{Hom}\left(G, \mathbf{C}^{*}\right) \mid H_{1}(X, \rho) \geq i\right\}
$$

and that $\rho=1$ belongs to $V_{\operatorname{dim} K e r \cup_{X}}$ (cf. [L3], Prop.1.1).

For example if $G=F_{r}$ then $\operatorname{dim}_{0}\left(F_{r}, \rho\right)$ is 0 , if $\rho$ is non trivial, and 1 otherwise. Using $e\left(F_{r}, \rho\right)=r-1$ we obtain that $\operatorname{dim} H^{1}\left(F_{r}, \rho\right)$ is $r-1$, if $\rho$ is non trivial, and otherwise is $r$. Since $\operatorname{dimKer} \cup_{F_{r}}=\left(\begin{array}{l}r \\ 2\end{array}\right)$ we recover the description of the characteristic varieties for $F_{r}$ mentioned in (1.2.2.1). 


\subsubsection{Structure of characteristic varieties.}

We will need the following theorem of D.Arapura (cf. [Ar]) which generalizes the results of C.Simpson to quasi-projective case.

Let $\bar{X}$ be a Kähler manifold such that $H^{1}(\bar{X}, \mathbf{C})=0, D$ a divisor with normal crossings and $X=\bar{X}-D$. Then, for each characteristic variety $V$, there exist a finite number of torsion characters $\rho_{i} \in \operatorname{Hom}\left(G, \mathbf{C}^{*}\right)$, a finite number of unitary characters $\rho_{j}^{\prime}$ and surjective maps onto (quasiprojective) curves $f_{i}: X \rightarrow C_{i}$ such that

$$
V(X)=\bigcup_{i} \rho_{i} f^{*} H^{1}\left(C_{i}, \mathbf{C}^{*}\right) \cup \bigcup \rho_{j}^{\prime}
$$

A consequence of 1.4.2.1 for curves in $\mathbf{C}^{2}$ is that the components of positive dimensions of their characteristic varieties are subtori of $\mathbf{C}^{* r}$ translated by points of finite order $\mathrm{t}$.

\subsubsection{Essential for a given set of components tori.}

By coordinate torus (corresponding to components $C_{i_{1}}, \ldots C_{i_{s}}$ ) we shall mean a subtorus in $\mathbf{C}^{* r}$ given by

$$
t_{i_{1}}=\ldots=t_{i_{s}}=1 \text {. }
$$

The inclusion $I_{i_{1}, . ., i_{s}}: \mathbf{C}^{2}-\cup_{i=1, \ldots, r} C_{i} \rightarrow \mathbf{C}^{2}-\cup_{i \neq i_{1}, \ldots, i_{s}} C_{i}$ induces a surjective map $\tilde{I}_{i_{1}, \ldots, i_{s}}: \pi_{1}\left(\mathbf{C}^{2}-\cup_{i=1, \ldots, r} C_{i}\right) \rightarrow \pi_{1}\left(\mathbf{C}^{2}-\cup_{i \neq i_{1}, \ldots, i_{s}} C_{i}\right)$ with restriction $\tilde{I}_{i_{1}, . ., i_{s}}^{\prime}: \pi_{1}^{\prime}\left(\mathbf{C}^{2}-\cup_{i=1, \ldots r} C_{i}\right) \rightarrow \pi_{1}^{\prime}\left(\mathbf{C}^{2}-\cup_{i \neq i_{1}, . . i_{s}} C_{i}\right)$ which is also surjective. Indeed if $K=\operatorname{Ker} \pi_{1}\left(\mathbf{C}^{2}-\cup_{i=1,, r} C_{i}\right) \rightarrow H_{1}\left(\mathbf{C}^{2}-\cup_{i \neq i_{1}, \ldots, i_{s}} C_{i}\right)$ then $K \rightarrow$ $\pi_{1}^{\prime}\left(\mathbf{C}^{2}-\cup C_{i \neq i_{1}, ., i_{s}}\right)$ is surjective. Since $K^{\prime}$ is a normal closure of $\pi_{1}^{\prime}\left(\mathbf{C}^{2}-\right.$ $\left.\cup_{i=1, \ldots r} C_{i}\right)$ and loops trivial in $\pi_{1}\left(\mathbf{C}^{2}-\cup_{i \neq i_{1}, \ldots, i_{s}} C_{i}\right.$ ) (e.g. loops which consist

\footnotetext{
${ }^{1}$ though the paper $[\mathrm{Ar}]$ considers only the case of the first characteristic variety (i.e. in terminology of $[\mathrm{Ar}]$ characters $\rho$ such that $\left.\operatorname{dim}^{1}(\rho) \geq 1\right)$, D.Arapura communicated to the author that the statement is true for all $V_{k}$. Moreover it follows from his argument that the dimension of $V_{k}$, containing the identity of the group of characters, is $k+1$. Indeed by (1.4.2.1) for any local system in such irreducible component of positive dimension of $V_{k}(X)$ there exist $L^{\prime}$ on an appropriate curve $C$ and the map $f: X \rightarrow C$ such that $L=f^{*} L^{\prime}$. Moreover it follows from Proposition 1.7 in [Ar] that for all but finitely many $L$ one has $H_{1}(X, L)=H_{1}\left(C, L^{\prime}\right)$. But $\pi_{1}(C)$ is free and if $k+1$ is the number of its generators and $L^{\prime}$ is not trivial then $\operatorname{dim}_{1}\left(C, L^{\prime}\right)=k$ (cf. 1.2.2.1).
} 
of paths from the base point to a point in vicinity of $C_{i},\left(i \neq i_{1}, . ., i_{s}\right)$ and loops bounding small disk transversal to $\left.C_{i}\right)$ the surjectivity of $I_{i_{1}, . ., i_{s}}^{\prime}$ follows.

The latter gives rise to a surjective map of $\mathbf{C}\left[H_{1}\left(\mathbf{C}^{2}-\cup_{i=1, ., r} C_{i}\right)\right]$-modules:

$\pi_{1}^{\prime} / \pi_{1}^{\prime \prime}\left(\mathbf{C}^{2}-\cup_{i=1, . ., r} C_{i}\right) \rightarrow \pi_{1}^{\prime} / \pi_{1}^{\prime \prime}\left(\mathbf{C}^{2}-\cup_{i \neq i_{1}, \ldots, i_{s}} C_{i}\right)$ which induces an injection of corresponding characteristic varieties:

$$
V_{k}\left(\mathbf{C}^{2}-\cup_{i \neq i_{1}, \ldots, i_{s}} C_{i}\right) \rightarrow V_{k}\left(\mathbf{C}^{2}-\cup_{i=1, . ., r} C_{i}\right)
$$

(cf. Lemma 1.2.1). A component of $V_{k}\left(\mathbf{C}^{2}-\cup_{i=1, ., r} C_{i}\right)$ which is an image of a component for some $i_{1}, \ldots, i_{s}$ in (1.4.3.2) is called obtained via a pull back. A component of $V_{k}\left(\mathbf{C}^{2}-\cup_{i=1, . ., r} C_{i}\right)$ is called essential if it isn't a pull back of component of a characteristic variety of a curve composed of irreducible components of $\mathcal{C}$.

Lemma 1.4.3 Let $V$ be a connected component of the characteristic variety $V_{1}$ of $\mathcal{C}$ having positive dimension and belonging to the coordinate torus $t_{i_{1}}=\ldots=t_{i_{s}}=1$. Then it is obtained via a pull back of a component of characteristic variety for the union of components of $\cup C_{i}\left(i \neq i_{1}, \ldots, i_{s}\right)$.

Proof. According to Arapura's theorem (cf. [Ar],(1.4.2)) component $V$ defines a map $f: \mathbf{C}^{2}-\mathcal{C} \rightarrow C$ for some quasiprojective curve $C$ such that for some local system $E \in \operatorname{Char}_{1}\left(\mathbf{C}^{2}-\mathcal{C}\right)$ one has: $V=E \otimes f^{*}(\operatorname{Char} C)$ where $\operatorname{Char} C=\operatorname{Hom}\left(\pi_{1}(C), \mathbf{C}^{*}\right)$. We claim that $f$ factors as follows:

$$
\begin{array}{cc}
\mathbf{C}^{2}-\mathcal{C} \underset{i_{1}, \ldots, i_{s}}{\longrightarrow} & \mathbf{C}^{2}-\cup_{i \neq i_{1}, \ldots, i_{s}} C_{i} \\
\searrow f & \downarrow \widetilde{f} \\
& C
\end{array}
$$

The lemma is a consequence of existence of $\tilde{f}$. Indeed for almost all local systems $L$ on $C$ we have $H^{1}\left(E \otimes f^{*}(L)\right)=H^{1}\left(f_{*}(E) \otimes L\right)$ (cf. proof of Prop. 1.7 in $[\mathrm{Ar}])$. Moreover $H^{1}\left(f_{*}(E) \otimes L\right)=H^{1}\left(\tilde{f}_{*} \circ\left(I_{i_{1}, \ldots, i_{s}}\right)_{*}(E) \otimes L\right)=$ $H^{1}\left(\left(I_{i_{1}, ., i_{s}}\right)_{*} E \otimes \tilde{f}^{*} L\right)$ and the latter has the same dimesnion for almost all $L$ again by the same argument from the proof of Prop. $1.7 \mathrm{in}[\mathrm{Ar}]$.

To show the existence of $\tilde{f}$, let $D=\bar{C}-C$ where $\bar{C}$ is a non singular compactification of $C$. Since for $j=i_{1}, . ., i_{s}$ we have $t_{j}=1$ on a translate (i.e. a coset) of $f^{*}\left(\operatorname{Hom}\left(H_{1}(C), \mathbf{C}^{*}\right)\right)$ and hence $t_{j}=1$ on the latter subgroup 
of $\operatorname{Char}\left(\pi_{1}\left(\mathbf{C}^{2}-\mathcal{C}\right)\right)$ we have for any $\chi \in \operatorname{Char}\left(H_{1}(C, \mathbf{Z})\right)$ and $j=i_{1}, . ., i_{s}$ the following: $\chi\left(f_{*}\left(\gamma_{j}\right)\right)=f^{*}(\chi)\left(\gamma_{j}\right)=t_{j}\left(f^{*}(\chi)\right)=1$. Equivalently $f_{*}\left(\gamma_{j}\right)=0$. Therefore $f_{*}(\gamma)=0$ in $H_{1}(C, \mathbf{Z})$ for $\gamma \in H_{1}\left(\mathbf{C}^{2}-\mathcal{C}, \mathbf{Z}\right)$ if and only if $\gamma$ belongs to the subgroup generated by $\gamma_{i_{1}}, \ldots, \gamma_{i_{s}}$. Let us consider the pencil of curves on $\mathbf{P}^{2}$ formed by the fibres of $f . f$ extends to the map from the complement to the base locus of this pencil to $\bar{C}$ ?. Preimage of $D$ in this extension is a union of components of $\mathcal{C}$ and we want to show that none of these components is $C_{i}$ with $i=i_{1}, \ldots i_{s}$. But none of the components $C_{i}, i=i_{1}, \ldots, i_{s}$ is taken by this extension into $D$ since otherwise $f_{*}\left(\gamma_{i}\right) \neq 0$ for the corresponding $\gamma_{i}$. Hence domain of this extension of $f$ contains all points of $C_{i_{1}} \cup \ldots \cup C_{i_{s}}$ not belonging to the remaining components of $\mathcal{C}$.

Note that for isolated points of characteristic varieties it can occur that $H^{1}\left(\mathbf{C}^{2}-\cup_{i \neq i_{1}, . ., i_{s}} C_{i}, L\right) \neq H^{1}\left(\mathbf{C}^{2}-C, I_{i_{1}, . ., i_{s}}^{*}(L)\right)$ as is shown by examples in [CS].

\subsection{Adjoints for complete intersections}

\section{5 .1}

Let $F \subset \mathbf{P}^{n}$ be a surface which is a complete intersection given by the equations:

$$
\bar{F}_{1}=\ldots=\bar{F}_{n-2}=0
$$

of degrees $d_{1}, \ldots, d_{n-2}$ respectively. Let (cf. [Ha] p.242)

$$
\left.\Omega_{F}=\mathcal{E} x t^{n-2}\left(\mathcal{O}_{F}, \Omega_{\mathbf{P}^{n}}^{n}\right)\right)
$$

be the dualizing sheaf of $F$. From the latter and the Koszul resolution

$0 \rightarrow \mathcal{O}_{\mathbf{P}^{n}}\left(-d_{1}-\ldots-d_{n-2}\right) \rightarrow \ldots . \rightarrow \mathcal{O}_{\mathbf{P}^{n}}\left(-d_{1}\right) \oplus \ldots \oplus \mathcal{O}_{\mathbf{P}^{n}}\left(-d_{n-2}\right) \rightarrow \mathcal{O}_{\mathbf{P}^{n}} \rightarrow \mathcal{O}_{F} \rightarrow 0$

it follows that one can identify $\Omega_{F}$ with $\mathcal{O}_{F}\left(d_{1}+\ldots+d_{n-2}-n-1\right)$.

Let $f: \tilde{F} \rightarrow F$ be a resolution of singularities of $F$ and $\tau: f_{*}\left(\Omega_{\tilde{F}}\right) \rightarrow \Omega_{F}$ be the trace map (cf. [BL]). It identifies sections of $f_{*}\left(\Omega_{\tilde{F}}\right)$ over an open set with those meromorphic differentials on non singular part of this open set in $F$ which when pulled back on a resolution $\tilde{F}$ admit a holomorphic extension

\footnotetext{
${ }^{2}$ incidentally, since the resolution $\bar{X}$ of the base locus of this pencil is simply-connected one has $\bar{C}=\mathbf{P}^{1}$
} 
over the exceptional set of $f$. The adjoint ideal $\mathcal{A}^{\prime}$ is the annihilator of the cokernel of $\tau$ i.e.

$$
\mathcal{A}^{\prime}=\mathcal{H o m}_{\mathcal{O}_{F}}\left(\Omega_{F}, f_{*}\left(\Omega_{\tilde{F}}\right)\right)=f_{*}\left(\Omega_{\tilde{F}}\right)\left(-d_{1}-\ldots-d_{n-2}+n+1\right)
$$

We define the sheaf of adjoint ideals on $\mathbf{P}^{n}$ as $\mathcal{A}=\pi^{-1}\left(\mathcal{A}^{\prime}\right)$ (also denoted as $A d j F$ ) where $\pi$ is the most right map in (1.5.1.2). The degeneration of Leray spectral sequence for $f$ (due to the Grauert-Riemenschneider vanishing theorem $([\mathrm{GR}]))$ yields

$$
\begin{gathered}
H^{i}\left(\tilde{F}, \Omega_{\tilde{F}}\right)=H^{i}\left(F, f_{*}\left(\Omega_{\tilde{F}}\right)\right)= \\
=H^{i}\left(F, \mathcal{A}^{\prime}\left(d_{1}+\ldots+d_{n-2}-n-1\right)=H^{i}\left(\mathbf{P}^{n}, \mathcal{A}\left(d_{1}+\ldots+d_{n-2}-n-1\right)\right)\right.
\end{gathered}
$$

In particular the irregularity of $\tilde{F}$ i.e. $\operatorname{dim} H^{1}(\tilde{F}, \mathcal{O})$ can be found as the difference between the actual dimension $H^{0}\left(\mathbf{P}^{n}, \mathcal{A}\left(d_{1}+\ldots+d_{n-2}-n-1\right)\right)$ and the "expected" dimension (i.e. $\left.\chi\left(\mathcal{A}\left(d_{1}+\ldots d_{n-2}-n-1\right)\right)\right)$ of the adjoints (since $H^{i}\left(\mathcal{A}\left(d_{1}+\ldots d_{n-2}-n-1\right)\right)=0$ for $\left.i \geq 2\right)$.

\subsubsection{Local description of adjoint ideals.}

Let

$$
F_{1}\left(w_{1}, \ldots, w_{n}\right)=0, \ldots, F_{n-2}\left(w_{1}, \ldots, w_{n}\right)=0
$$

be a germ of a complete intersection of hypersurfaces in $\mathbf{C}^{n}$ having an isolated singularity at the origin $O$. For any two pairs $1 \leq i, j \leq n, i \neq j$ and $1 \leq k, l \leq n, k \neq l$ we have up to sign:

$$
\frac{d w_{i} \wedge d w_{j}}{\frac{\partial\left(F_{1}, \ldots, F_{n-2}\right)}{\partial\left(w_{1}, \ldots, \hat{w}_{i}, . ., \hat{w}_{j}, . ., w_{n}\right)}}=\frac{d w_{k} \wedge d w_{l}}{\frac{\partial\left(F_{1}, \ldots, F_{n-2}\right)}{\partial\left(w_{1}, \ldots, \hat{w}_{k}, \ldots, \hat{w}_{l}, \ldots, w_{n}\right)}}
$$

Indeed the Cramer's rule for the solutions of the system of equations:

$$
\frac{\partial F_{k}}{\partial w_{1}} d w_{1} \wedge d w_{i}+\ldots+\frac{\partial F_{k}}{\partial w_{n}} d w_{n} \wedge d w_{i}=0 \quad(k=1, \ldots, n-2)
$$

when one views $d w_{k} \wedge d w_{i}(k=1, . ., \hat{i}, ., n-1)$ as unknowns yields that up to sign:

$$
d w_{k} \wedge d w_{i}=\frac{\frac{\partial\left(F_{1}, \ldots, F_{n-2}\right)}{\partial\left(w_{1}, \ldots, \hat{w}_{k}, \ldots, \hat{w}_{i}, \ldots, w_{n}\right)} d w_{n} \wedge d w_{i}}{\frac{\partial\left(F_{1}, \ldots, F_{n-2}\right)}{\partial\left(w_{1}, \ldots, \hat{w}_{i}, \ldots, w_{n-1}\right)}}
$$


(1.5.2.2) follows from this for any two pairs $(i, j),(k, l), i \neq j, k \neq l$.

Since $F_{1}=\ldots=F_{n-2}=0$ is a complete intersection with isolated singularity one of the Jacobians $\frac{\partial\left(F_{1}, . ., F_{n-2}\right)}{\partial\left(w_{1}, ., \hat{w}_{i}, \ldots, \hat{w}_{j}, \ldots, w_{n}\right)}$ is non vanishing in a neighborhood of the singularity everywhere except for the singularity itself. In particular each side (1.5.2.2) defines a holomorphic 2-form outside of the origin for any $(i, j), i \neq j$ or $(k, l), k \neq l$. In fact this form is just the residue of the $\log$-form $\frac{d z_{1} \wedge \ldots \wedge d z_{n}}{F_{1} \cdots F_{n-2}}$ at non singular points (i.e. outside of the origin) of (1.5.2.1).

The adjoint ideal $\mathcal{A}_{O}$ in the local ring $\mathcal{O}_{O}$ of the origin of a germ of complete intersection (1.5.2.1), according to the description of the trace map 1.5.1 can be made explicit as follows. Let $f: \tilde{\mathbf{C}}^{n} \rightarrow \mathbf{C}^{n}$ be an embedded resolution of (1.5.1.1). Then $\mathcal{A}_{O}$ consists of $\phi \in \mathcal{O}_{O}$ such that $f^{*}\left(\phi \cdot \frac{d w_{i} \wedge d w_{j}}{\frac{\partial\left(F_{1}, \ldots, F_{n-2}\right)}{\partial\left(w_{1}, \ldots, \hat{w}_{i}, \ldots, \hat{w}_{j}, \ldots, w_{n}\right)}}\right)$ admits a holomorphic extension from $f^{-1}\left(\mathbf{C}^{n}-O\right)$ to $\tilde{\mathbf{C}}^{n}$.

Similarly, the elements of $H^{0}\left(\mathcal{A}\left(d_{1}+\ldots+d_{n-2}-n-1\right) \subset H^{0}\left(\Omega_{\mathbf{P}^{n}}\left(d_{1}+\right.\right.\right.$ $\left.\left.\ldots+d_{n-2}\right)\right)$ can be viewed as meromorphic forms with log singularities near non singular points of (1.5.1.1) having as residue a 2 -form on a non singular locus of $F$ and admitting a holomorphic extension on $\tilde{F}$.

\section{Ideals and polytopes of quasiadjunction.}

\subsection{Ideals of quasiadjunction.}

Let $f$ be a germ of a reduced algebraic curve having a singularity with $r$ irreducible branches at the origin of $\mathbf{C}^{2}$ near which it is given by local equation $f=f_{1}(x, y) \cdots f_{r}(x, y)=0$. Let $\mathcal{O}$ be the local ring of the origin and $A$ be an ideal in $\mathcal{O}$.

Definition 2.1.1 An ideal $A$ is called an ideal of quasiadjunction of $f$ with parameters $\left(j_{1}, . ., j_{r} \mid m_{1}, \ldots, m_{r}\right)\left(j_{i}, m_{i}\right.$ are integers $)$ if $A=\left\{\phi \in \mathcal{O} \mid z_{1}^{j_{1}} \ldots z_{r}^{j_{r}} \phi \in\right.$ $\left.\operatorname{Adj} V_{\left(m_{1}, f_{1}\right), \ldots,\left(m_{r}, f_{r}\right)}\right\}$ where $V_{\left(m_{1}, f_{1}\right),,,\left(m_{r}, f_{r}\right)}$ is a germ at the origin of the complete intersection in $\mathbf{C}^{r+2}$ given by the equations:

$$
z_{1}^{m_{1}}=f_{1}(x, y), \ldots, z_{r}^{m_{r}}=f_{r}(x, y) .
$$

An ideal of quasiadjunction is an ideal in $\mathcal{O}$ which is an ideal of quasiadjunction for some system of parameters. 


\subsection{Basic Ideal.}

Let $A\left(f_{1}, \ldots, f_{r}\right) \subset \mathcal{O}$ be the ideal generated by

$\frac{\left(f_{i}\right)_{x}}{f_{i}} f_{1} \cdot f_{2} \cdots f_{r}, \frac{\left(f_{i}\right)_{y}}{f_{i}} f_{1} \cdot f_{2} \cdots f_{r},(i=1, . ., r), \frac{\left.\operatorname{Jac}\left(\frac{\left(f_{i}, f_{j}\right)}{(x, y)}\right)\right)}{f_{i} f_{j}} f_{1} \cdots f_{r},(i, j=1, \ldots, r, i \neq j)$

(we shall call it the basic ideal).

Equating all polynomials (2.2.1) to zero yields a system of equations having $(0,0)$ as the only solution. Therefore $\mathcal{O} / A\left(f_{1}, \ldots, f_{r}\right)$ is an Artinian algebra.

Moreover for any set of parameters $\left(i_{1}, \ldots, i_{r} \mid m_{1}, \ldots, m_{r}\right)$ the corresponding ideal of quasiadjuction contains $A\left(f_{1}, \ldots, f_{r}\right)$. Indeed, if $F_{i}=z_{i}^{m_{i}}-f_{i}(x, y)$, then up to $\operatorname{sign} \frac{\partial\left(F_{1}, \ldots, F_{r}\right)}{\partial\left(z_{1}, \ldots, \hat{z}_{i}, . ., z_{r}, x\right)}=z_{1}^{m_{1}-1} \cdots z_{i}^{\hat{m_{i}-1}} \cdots z_{r}^{m_{r}-1} \cdot\left(f_{i}\right)_{x}$ and hence

$\frac{\left(f_{i}\right)_{x} f_{1} \cdots \hat{f}_{i} \cdots f_{r} d z_{i} \wedge d y}{\frac{\partial\left(F_{1}, \ldots F_{r}\right)}{\partial\left(z_{1}, . . \hat{z}_{i}, \ldots, z_{r}, x\right)}}=\frac{\left(f_{i}\right)_{x} f_{1} \cdots \hat{f}_{i} \cdots f_{r} d z_{i} \wedge d x}{z_{1}^{m_{1}-1} \cdots \hat{z}_{i}^{m_{i}-1} \cdots z_{r}^{m_{r}-1}\left(f_{i}\right)_{x}}=z_{1} \cdots \hat{z}_{i} \cdots z_{r} d z_{i} \wedge d y$

which is holomorphic on $\mathbf{C}^{r+2}$. Similarly, one sees that the 2-forms corresponding to other generators of $A\left(f_{1}, . ., f_{r}\right)$ coincide on $F_{1}=\ldots=F_{r}=0$ with the forms admitting a holomorphic extension to $\mathbf{C}^{r+2}$.

In particular, there are only finitely many ideals of quasiadjunction.

\subsection{Ideals of quasiadjunction and polytopes.}

Let $\mathcal{U}=\left\{\left(x_{1}, . ., x_{r}\right) \in \mathbf{R}^{r} \mid 0 \leq x_{i}<1\right\}$ be the unit cube with coordinates corresponding to the components of a curve $\mathcal{C}$. Sometimes we shall denote this cube as $\mathcal{U}(\mathcal{C})$. If $\mathcal{C}^{\prime}$ is formed by components of $\mathcal{C}$ then we shall view $\mathcal{U}\left(\mathcal{C}^{\prime}\right)$ as the face of $\mathcal{U}(\mathcal{C})$ given by $x_{j}=0$ where $j$ runs through indices corresponding to components of $\mathcal{C}$ not belonging to $\mathcal{C}^{\prime}$.

By a convex polytope mean a subset of $\mathbf{R}^{n}$ [ which is the convex hull of a finite set of points with some faces possibly deleted. By a polytope we mean a finite union of convex polytopes. Class of polytopes in this sense is closed under finite unions and intersections. A complement to a polytope within an ambient polytope is a polytope. By face of maximal dimension

\footnotetext{
${ }^{3}$ only subsets of $\mathcal{U}$ will occur below.
} 
of a polytope we mean the intersection of the polytope's boundary with the hyperplane for which this intersection has the dimension equal to the dimension of the boundary. A face of a polytope is an intersection of faces of maximal dimension. This is again a closed polytope.

Each $\left(x_{1}, \ldots, x_{r}\right) \in \mathcal{U}$ defines the character $\chi\left(x_{1},,,,, x_{r}\right)$ of $\pi_{1}\left(\mathbf{C}^{2}-\mathcal{C}\right)$ such that $\chi\left(x_{1}, \ldots, x_{r}\right)\left(\gamma_{i}\right)=\exp \left(2 \pi \sqrt{-1} x_{i}\right)$. We shall call it the exponential map. We also put $\overline{\left(x_{1}, \ldots, x_{r}\right)}=\left(1-x_{1}, \ldots, 1-x_{r}\right)$ and call this conjugation since $\chi\left(1-x_{1}, \ldots, 1-x_{r}\right)=\overline{\chi\left(x_{1}, \ldots, x_{r}\right)}$. This map is an involution of the interior of $\mathcal{U}: \mathcal{U}^{\circ}=\left\{\left(x_{1}, \ldots, x_{r}\right) \in \mathcal{U} \mid x_{i} \neq 0, i=1, \ldots r\right\}$.

Different arrays $\left(i_{1}, \ldots, i_{r} \mid m_{1}, \ldots, m_{r}\right)$ may define the same ideals of quasiadjunction. The next proposition describes when this is the case.

Proposition 2.3.1 Let $A$ be an ideal of quasiadjunction. Then there is a polytope $\bar{\Delta}(A)$, which is a open subset in $\mathcal{U}$, with the following property: for $\left(m_{1}, \ldots, m_{r}\right) \in \mathbf{Z}^{r}$ and $\left(j_{1}, \ldots, j_{r}\right) \in \mathbf{Z}^{r}, 0 \leq j_{i}<m_{i}, i=1, \ldots, r$ a holomorphic function $z_{1}^{j_{1}} \cdots z_{r}^{j_{r}} \phi$ belongs to the adjoint ideal of the germ of an abelian branched cover of the type $\left(m_{1}, . ., m_{r}\right)$ of a neighborhood of the origin in $\mathbf{C}^{2}$ for any $\phi \in A$ if and only if $\left(\frac{j_{1}+1}{m_{1}}, \ldots, \frac{j_{r}+1}{m_{r}}\right) \in \bar{\Delta}(A)$.

Proof. Let $\rho: Y_{f} \rightarrow \mathbf{C}^{2}$ be an embedded resolution for the singularity $f=$ $f_{1}(x, y) \cdots f_{r}(x, y)=0$ at the origin. The complete intersection $V_{\left(m_{1}, f_{1}\right), \ldots,\left(m_{r}, f_{r}\right)}$ (cf. (2.1.1)) provides a model with an isolated singularity of a branched abelian cover of a neighborhood of the origin in $\mathbf{C}^{2}$ with $f=0$ as its branching locus. Let $\pi_{\left(m_{1}, f_{1}\right), \ldots,\left(m_{r}, f_{r}\right)}: V_{m_{1}, \ldots, m_{r}} \rightarrow \mathbf{C}^{2}$ be the canonical projection. If $\bar{V}_{\left(m_{1}, f_{1}\right), \ldots,\left(m_{r}, f_{r}\right)}$ is the normalization of $Y_{f} \times_{\mathbf{C}^{2}} V_{\left(m_{1}, f_{1}\right) \ldots,\left(m_{r}, f_{r}\right)}$ then the projection $\rho_{\left(m_{1}, f_{1}\right) \ldots,\left(m_{r}, f_{r}\right)}: \bar{V}_{\left(m_{1}, f_{1}\right), \ldots,\left(m_{r}, f_{r}\right)} \rightarrow V_{\left(m_{1}, f_{1}\right) \ldots,\left(m_{r}, f_{r}\right)}$ on the second factor is a resolution of the singularity at the origin in the category of $V$ manifolds. We have the diagram:

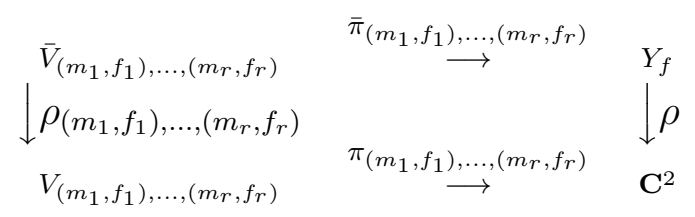

Let $E=\cup_{k} E_{k}$ be the exceptional locus of $\rho$. The exceptional locus of $\rho_{\left(m_{1}, f_{1}\right), \ldots,,\left(m_{r}, f_{r}\right)}$ is $\cup E_{k, l}$ where $E_{k, l}$ is a cover of $E_{k}$.

Let $a_{k, i}=$ mult $_{E_{k}} \rho^{*} f_{i}(x, y),(i=1, \ldots, r), c_{k}=$ mult $_{E_{k}} \rho^{*}(d x \wedge d y)$. For $\phi \in \mathcal{O}$ we put $f_{k}(\phi)=$ mult $_{E_{k}} \rho^{*}(\phi)$. Finally let $g_{k, i}=\operatorname{g.c.d}\left(m_{i}, a_{k, i}\right),(i=$ 
$1, \ldots, r)$ and $s_{k}=$ g.c.d. $\left(\frac{m_{1}}{g_{k, 1}}, \ldots \frac{m_{r}}{g_{k, r}}\right),(i=1, \ldots, r)$. The form $\frac{d x \wedge d y}{z_{1}^{m_{1}-1} \ldots z_{r}^{m^{-1}}}$ is a non vanishing form on

$$
V_{\left(m_{1}, f_{1}\right), . .,\left(m_{r}, f_{r}\right)}-\operatorname{Sing} V_{\left(m_{1}, f_{1}\right), \ldots,\left(m_{r}, f_{r}\right)}
$$

(cf. (1.5.2.2)). If $A$ is an ideal of quasi-adjunction with parameters $\left(\bar{j}_{1}, \ldots, \bar{j}_{r} \mid\right.$ $\left.m_{1}, \ldots, m_{r}\right)$ then the condition $\phi \in A$ is equivalent to the existence of a holomorphic extension of $\rho_{\left(m_{1}, f_{1}\right), \ldots,\left(m_{r}, f_{r}\right)}^{*}\left(\frac{z_{1}^{\bar{j}_{1}} \ldots z_{r}^{\bar{j}_{r}} \phi d x \wedge d y}{z_{1}^{m_{1}-1} \ldots z_{r}^{m_{r}-1}}\right)$ over the exceptional locus $\cup E_{k, l}$ in a neighborhood of each point of $\cup E_{k, l}$ not belonging to $E_{k, l} \cap E_{\bar{k}, \bar{l}}$ for any $(k, l),(\bar{k}, \bar{l})$. This, in turn, is equivalent to:

$$
\begin{gathered}
\sum_{i=1}^{i=r}\left(\bar{j}_{i}-m_{i}+1\right) \text { mult }_{E_{k, l}} \rho_{\left(m_{1}, f_{1}\right), \ldots,\left(m_{r}, f_{r}\right)}^{*}\left(z_{j}\right)+\text { mult }_{E_{k, l}} \rho_{\left(m_{1}, f_{1}\right), \ldots,\left(m_{r}, f_{r}\right)}^{*} \phi+ \\
\left.+ \text { mult }_{E_{k, l}} \rho_{\left(m_{1}, f_{1}\right), \ldots,\left(m_{r}, f_{r}\right)}^{*}(d x \wedge d y)\right) \geq 0
\end{gathered}
$$

for any pair of indices $(k, l)$. On the other hand, we have the following equalities:

$$
\begin{gathered}
\operatorname{mult}_{E_{k, l}} \rho_{\left(m_{1}, f_{1}\right), \ldots,\left(m_{r}, f_{r}\right)}^{*}\left(z_{i}\right)=\frac{m_{1} \cdots \hat{m}_{i} \cdots m_{r} \cdot a_{k, i}}{g_{k, 1} \cdots g_{k, r} \cdot s_{k}}, \operatorname{mult}_{E_{k, l}} \rho_{\left(m_{1}, f_{1}\right), \ldots,\left(m_{r}, f_{r}\right)}^{*}(\phi)= \\
=\frac{f_{k}(\phi) \cdot m_{1} \cdots m_{r}}{g_{k, 1} \cdots g_{k, r} \cdot s_{k}}, \\
\left.\operatorname{mult}_{E_{k, l} \rho_{\left(m_{1}, f_{1}\right), \ldots,\left(m_{r}, f_{r}\right)}^{*}(d x} \wedge d y\right)=\frac{c_{k} \cdot m_{1} \cdots m_{r}}{g_{k, 1} \cdots g_{k, r} \cdot s_{k}}+\frac{m_{1} \cdots m_{r}}{g_{k, 1} \cdots g_{k, r} \cdot s_{k}}-1
\end{gathered}
$$

To see (2.3.3), we can select local coordinates $(u, v)$ on $Y_{f}$ near a point belonging to a single component $E_{k}$ in which the latter is given by the equation $u=0$. Then $\rho^{*}\left(f_{i}(x, y)\right)=u^{a_{k, i}} \cdot \epsilon_{i}(u, v)$ where $\epsilon_{i}(u, v)(i=1, \ldots, r)$ are units in the corresponding local ring. The fiber product $Y_{f} \times_{\mathbf{C}^{2}} V_{\left(m_{1}, f_{1}\right), . .,\left(m_{r}, f_{r}\right)}$ is a subvariety in $\mathbf{C}^{r+2} \times \mathbf{C}^{2} Y_{f}$ given by the equations:

$$
z_{1}^{m_{1}}=u^{a_{k, 1}} \epsilon_{1}(u, v), \ldots, z_{r}^{m_{r}}=u^{a_{k, r}} \epsilon_{r}(u, v)
$$

Each branch of (2.3.4) has the following local parameterization:

$$
u=t^{\frac{m_{1} \cdots m_{r}}{g_{k, 1} \cdots g_{k, r} \cdot s_{k}}}, z_{i}=t^{\frac{m_{1} \cdots \hat{m}_{i} \cdots m_{r} \cdot a_{k, i}}{g_{k, 1} \cdots g_{k, r} \cdot s_{k}}}, i=1, \ldots, r
$$


(exponents are chosen so that their greatest common divisor will be equal to 1 and so that they will satisfy (2.3.4)). This yields the first equality in (2.3.3). We have

$$
\operatorname{mult}_{E_{k, l}} \rho_{\left(m_{1}, f_{1}\right), \ldots,\left(m_{r}, f_{r}\right)}^{*}(\phi)=\operatorname{mult}_{E_{k, l}} \bar{\pi}_{\left(m_{1}, f_{1}\right), \ldots,\left(m_{r}, f_{r}\right)} u^{f_{k}(\phi)} .
$$

Hence the second equality in (2.3.3) follows from (2.3.5).

Finally, since the map $\bar{\pi}_{\left(m_{1}, f_{1}\right), \ldots,\left(m_{r}, f_{r}\right)}$ is given locally by $(t, v) \rightarrow\left(t^{\frac{m_{1} \cdots m_{r}}{g_{k, 1} \cdots g_{k, r}}}, v\right)$, we have:

$$
\begin{gathered}
\rho_{\left(m_{1}, f_{1}\right), \ldots,\left(m_{r}, f_{r}\right)}^{*}\left(d x \wedge d y \mid V_{\left(m_{1}, f_{1}\right), \ldots,\left(m_{r}, f_{r}\right)}\right)= \\
=\rho_{\left(m_{1}, f_{1}\right), \ldots,\left(m_{r}, f_{r}\right)}^{*} \circ \pi_{\left(m_{1}, f_{1}\right), \ldots,\left(m_{r}, f_{r}\right)}^{*}\left(d x \wedge d y \mid \mathbf{C}^{2}\right)= \\
=\bar{\pi}_{\left(m_{1}, f_{1}\right), \ldots,\left(m_{r}, f_{r}\right)}^{*}\left(u^{c_{k}} d u \wedge d v\right)=t^{\frac{c_{k} \cdot m_{1} \cdots m_{r}}{g_{k, 1} \cdots g_{k, 1} \cdot s_{k}}+\frac{m_{1} \cdots m_{r}}{g_{k, 1} \cdots g_{k, r} \cdot s_{k}}-1} d t \wedge d v
\end{gathered}
$$

which implies the last equality in (2.3.3).

Now it follows from $(2.3 .2)$ and $(2.3 .5)$ that $\phi \in A\left(\bar{j}_{1}, \ldots, \bar{j}_{r} \mid m_{1}, \ldots, m_{r}\right)$ if and only if for any $k$ the multiplicity $f_{k}(\phi)$ satisfies:

$$
\begin{gathered}
\sum_{i=1}^{i=r}\left(\bar{j}_{i}-m_{i}+1\right) \frac{m_{1} \cdots \hat{m}_{i} \cdots m_{r} \cdot a_{k, i}}{g_{k, 1} \cdots g_{k, r} s_{k}}+\frac{m_{1} \cdots m_{r} \cdot f_{k}(\phi)}{g_{k, 1} \cdots g_{k, r} \cdot s_{k}}+ \\
+\frac{c_{k} \cdot m_{1} \cdots m_{r}}{g_{k, 1} \cdots g_{k, r} \cdot s_{k}}+\frac{m_{1} \cdots m_{r}}{g_{k, 1} \cdots g_{k, r} \cdot s_{k}}-1 \geq 0
\end{gathered}
$$

For given $k$ let $f_{k}(A)$ be the minimal integer solution, with $f_{k}(\phi)$ considered as unknown, for this inequality and $\phi_{k}$ be such that $f_{k}\left(\phi_{k}\right)=f_{k}(A)$. In other words $\phi \in A$ if and only if $f_{k}(\phi) \geq f_{k}(A)$. We have $f_{k}(A)=\left\{\sum_{i=1}^{i=k}\left(a_{k, i}-\left(\bar{j}_{i}+\right.\right.\right.$ 1) $\left.\left.\frac{a_{k, i}}{m_{i}}\right)-c_{k}-1\right\}=\left[\left(\sum_{i=1}^{i=k}\left(a_{k, i}-\frac{\bar{j}_{i}+1}{m_{i}} a_{k, i}\right)-c_{k}\right]\right.$ where $\{r\}$ (resp. $\left.[r]\right)$ denotes the smallest integer which is strictly greater than (resp. the integer part of) $r$. We shall call $f_{k}(A)$ the multiplicity of $A$ along $E_{k}$. This is the minimum of multiplicities along $E_{k}$ of pull backs on $Y_{f}$ of elements of $A$.

The same calculation shows that $z_{1}^{j_{1}} \cdots z_{r}^{j_{r}} \phi$, where $\phi$ belongs to an ideal of quasiadjunction $A$, is in the adjoint ideal of $z_{1}^{m_{1}}=f_{1}(x, y), \ldots, z_{r}^{m_{r}}=f_{r}(x, y)$ if and only if:

$$
\begin{gathered}
\sum_{i=1}^{i=r}\left(j_{i}-m_{i}+1\right) \frac{m_{1} \cdots m_{r} a_{k}}{g_{k, 1} \cdots g_{k, r} \cdot m_{i} \cdot s_{k}}+\frac{m_{1} \cdots m_{r} f_{k}(A)}{g_{k, 1} \cdots g_{k, r} \cdot s_{k}}+ \\
+\frac{c_{k} \cdot m_{1} \cdots m_{r}}{g_{k, 1} \cdots g_{k, r} \cdot s_{k}}+\frac{m_{1} \cdots m_{r}}{g_{k, 1} \cdots g_{k, r} s_{k}}-1 \geq 0
\end{gathered}
$$


or equivalently:

$$
\sum_{i=1}^{i=r} \frac{j_{i}+1}{m_{i}} a_{k, i}>\sum_{i=1}^{i=r} a_{k, i}-f_{k}(A)-c_{k}-1
$$

Indeed, if (2.3.7) holds, then, since $f_{k}(\phi) \geq f_{k}(A)$ for any $\phi \in A$, one can replace $f_{k}(A)$ in $(2.3 .7)$ by $f_{k}(\phi)$. This converts (2.3.7) into a necessary and sufficient condition for $z_{1}^{j_{1}} \cdots z_{r}^{j_{r}} \phi$ to belong to the adjoint ideal of (2.1.1) (cf. derivation of (2.3.6)). Vice versa, for $\phi_{k}$ satisfying $f_{k}\left(\phi_{k}\right)=f_{k}(A)$, the condition that $z_{1}^{j_{1}} \cdots z_{r}^{j_{r}} \phi_{k}$ is in the adjoint ideal of (2.1.1), is nothing else but (2.3.7).

The polytope $\bar{\Delta}(A)$ satisfying the conditions of the proposition is the set of solutions of the inequalities:

$$
\sum_{i=1}^{i=r} x_{i} a_{k, i}>\sum_{i=1}^{i=r} a_{k, i}-f_{k}(A)-c_{k}-1
$$

for all $k$.

\subsubsection{Remarks.}

1. If $A_{1}$ and $A_{2}$ are ideals of quasiadjunction and $A_{1} \subset A_{2}$ then $\bar{\Delta}\left(A_{2}\right) \subset$ $\bar{\Delta}\left(A_{1}\right)$

2.The polytope corresponding the basic ideal $A\left(f_{1}, . ., f_{r}\right)$ (cf. 2.2) is the whole unit cube $\mathcal{U}$.

3. $\mathcal{O}$ is considered as improper "ideal" of quasiadjunction since $A\left(m_{1}-\right.$ $\left.1, \ldots, m_{r}-1 \mid m_{1}, . ., m_{r}\right)=\mathcal{O}$.

\subsection{Local polytopes of quasiadjunction and faces of quasiadjunction.}

\section{4 .1}

Definition 2.4.1 We say that two points in the unit cube $\mathcal{U}$ are equivalent if the collections of polytopes $\bar{\Delta}(A)$ containing each of the points coincide. A (local) polytope of quasiadjunction $\Delta$ is an equivalence class of points with this equivalence relation. 
Definition 2.4.2 A face of quasiadjunction is an intersection of a face ( $c f$. (2.3)) of a local polytope of quasiadjunction and a (different) polytope of quasiadjunction. In particular, each face of quasiadjunction belongs to a unique polytope of quasiadjunction.

For each face let us consider the system of equation defining the affine subspace of $\mathbf{Q}^{r}$ spanned by this face. One can normalize the system so that all coefficients of variables and the free term are integers and the g.c.d. of non zero minors of maximal order is equal to 1.

Definition 2.4.3 The order of a face of quasiadjunction is the g.c.d of minors of maximal order in the matrix of coefficients in the normalized system of linear equations defining this face.

This is the order of the torsion of the quotient of $\mathbf{Z}^{r}$ by the subgroup generated by the vectors having as coordinates the coefficients of variables in the equations of the face. In particular this integer is independent of the chosen normalized system of equations and depends only on the face of quasiadjunction.

The (local) ideal of quasiadjunction corresponding to a face of quasiadjunction is the ideal of quasiadjunction corresponding to the polytope of quasiadjunction containing this face. The ideal corresponding to a face of quasiadjunction has the form $A\left(j_{1}, \ldots, j_{r} \mid m_{1}, \ldots, m_{r}\right)$ where $\left(\frac{j_{1}+1}{m_{1}}, \ldots, \frac{j_{r}+1}{m_{r}}\right)$ belongs to this face.

\subsection{Examples.}

1. In the case of the branch with one component the ideals of quasiadjunction correspond to the constants of quasiadjunction (cf. [L2]). Recall that for a germ $\phi$ the rational number $\kappa_{\phi}$ is characterized by the property $\min \left\{i \mid z^{i} \phi \in\right.$ $\left.\operatorname{Adj}\left(z^{n}=f(x, y)\right)\right\}=\left[\kappa_{\phi} \cdot n\right]$. The ideal of quasiadjunction corresponding to $\kappa$ consists of $\phi$ such that $\kappa_{\phi}>\kappa$. For example for the cusp $x^{2}+y^{3}$ the only non zero constant of quasiadjunction is $\frac{1}{6}$, there are two polytopes of quasiadjunction i.e. $\Delta^{\prime}=\left\{x \in[0,1] \mid 1>x>\frac{1}{6}\right\}$ and $\Delta^{\prime \prime}=\{x \in$ $\left.[0,1] \mid \frac{1}{6} \geq x \geq 0\right\} . x=\frac{1}{6}$ is the face of quasiadjunction and the corresponding ideal of quasiadjunction is the maximal ideal. For an arbitrary unibranched 
singularity we have $\bar{\Delta}\left(A_{\kappa}\right)=\{x \mid x>\kappa\}$. The order of the face $x=\kappa$ is equal to the order of the root of unity $\exp (2 \pi i \kappa)$.

2. Let us consider a tacnode, locally given by the equation: $y\left(y-x^{2}\right)$ (i.e. $\left.f(x, y)=y, g(x, y)=y-x^{2}\right)$. Then the basic ideal is the maximal ideal and hence there is only one ideal of quasiadjunction. If $\mathcal{M}$ is the maximal ideal, then $\bar{\Delta}(\mathcal{M})$ is the whole unit square. To determine the polytope $\bar{\Delta}(\mathcal{O})$, note that after two blowups we obtain an embedded resolution which in one of the charts looks like: $x=u v, y=u^{2} v$ where $u=0$ and $v=0$ are the exceptional curves. Hence for the component $u=0$ we obtain $a=b=$ $2, f(\phi=1)=0, c=2$ and the corresponding polytope is $x+y>\frac{1}{2}$. The face of quasiadjunction is $x+y=\frac{1}{2}$ and the corresponding ideal is the maximal one.

3. For the ordinary singularity of multiplicity $m:\left(\alpha_{1} x+\beta_{1} y\right) \cdots\left(\alpha_{m} x+\right.$ $\left.\beta_{m} y\right)=0$ the basic ideal is $\mathcal{M}^{m-2}$ where $\mathcal{M} \subset \mathcal{O}$ is the maximal ideal of the local ring at the origin. Since the resolution can be obtained by a single blow up, we have $a_{1, i}=1, c_{1}=1, i=1, \ldots, m$ i.e. the polytope $\bar{\Delta}(A)$ of an ideal of quasiadjunction $A$ is:

$$
x_{1}+\ldots+x_{m}>m-2-f_{1}(A)
$$

Since $f_{1}(\phi) \geq f_{1}(A)$ is equivalent to $\phi \in \mathcal{M}^{f_{1}(A)}$ i.e. the latter is the ideal corresponding to the polytope (2.5.1). The faces of quasiadjunction are $x_{1}+$ $\ldots+x_{m}=m-2-f_{1}(A)\left(f_{1}(A)=0, \ldots, m-3\right)$ and the corresponding ideal of quasiadjunction is $\mathcal{M}^{f_{1}(A)+1}$.

Additional examples are discussed in [L7].

\subsection{Global polytopes and sheaves of quasiadjunction.}

Let $\mathbf{R}^{r}$, as in 2.3 , be vector space coordinates of which are in one to one correspondence with the components of the curve $\mathcal{C}=\cup_{i=1}^{i=r} C_{i}$. For a singular point $p$ of $\mathcal{C}$, let $\mathcal{C}_{p}$ be the collection of components of $C$ passing through $p$. Each polytope of quasiadjunction $\Delta_{p} \subset \mathcal{U}\left(\mathcal{C}_{p}\right)$ of $p$ defines the polytope in $\mathcal{U}=\left\{\left(x_{1}, . ., x_{r}\right) \mid 0 \leq x_{i} \leq 1\right\} \subset \mathbf{R}^{r}$ consisting of points $\left\{\left(x_{1}, \ldots, x_{r}\right) \mid\left(x_{1}, . ., x_{r}\right) \in \mathcal{U},\left(x_{i_{1}}, \ldots, x_{i_{r(p)}}\right) \in \Delta_{p}\right\}$ where $\left(i_{1}, \ldots, i_{r(p)}\right)$ are the coordinates corresponding to the components of $\mathcal{C}_{p}$ (i.e. passing through the singularity $p$ ). We shall use the notation $\Delta_{p}(\mathcal{U})$ for this polytope in $\mathcal{U}$. 


\subsubsection{Definition}

A type of a point in $\mathcal{U}$ is the collection of polytopes $\Delta_{p}(\mathcal{U}) \subset \mathcal{U}$ to which this point belongs, where $p$ runs through all $p \in \operatorname{Sing} \mathcal{C}$.

We call two points in $\mathcal{U}$ equivalent if they have the same type. A (global) polytope of quasiadjunction is an equivalence class of this equivalence relation. Global polytopes of quasiadjunction form a partition which is a refinement of every partitions of $\mathcal{U}$ defined by polytopes $\Delta_{p}(\mathcal{U})$ corresponding to local polytopes of quasiadjunction of singularities of $\mathcal{C}$.

\subsubsection{Definition.}

We shall call a face $\delta$ of quasiadjunction contributing if it belongs to a hyperplane $d_{1} x_{1}+\ldots+d_{r} x_{r}=l$ where $d_{1}, \ldots d_{r}$ are the degrees of the components of $\mathcal{C}$ corresponding to respective coordinates $x_{1}, \ldots, x_{r}$. This hyperplane is called contributing, the integer $l=l(\delta)$ is called the level of both the contributing hyperplane and contributing face. The order of a global face of quasiadjunction is defined as in local case (cf. 2.4.2). A polytope of quasiadjunction is called contributing if it contains a contributing face.

A point $\left(x_{1}, . ., x_{r}\right) \in \delta$ is called interior $\mathcal{C}^{\prime}$-point for a curve $\mathcal{C}^{\prime}$ formed by components of $\mathcal{C}$ if $x_{i} \neq 1$ if and only if $i$ corresponds to a component of $\mathcal{C}^{\prime}$ and $\left(x_{1}, . ., x_{r}\right)$ is in the interior of $\delta$.

Remarks 2.6.2.1 In the case when $r=1$, e.g. for an irreducible curve, an order of a global face of quasiadjunction is the order of a root of the Alexander polynomial. Indeed, for a constant of quasiadjunction $\kappa, \exp (2 \pi i \kappa)$ is a root of Alexander polynomial (cf. [LV]).

2.6.2.2. The collection of orders of faces of quasiadjunction for reducible curves a priori cannot be determined just by the local types of all singular points. However it is combinatorial invariant of the curve in the sense that it depends only on local information about singularities and specification of components which contain specified singular points, e.g. it is independent of the geometry of the set of singular points in $\mathbf{P}^{2}$. In the case of arrangements of lines this is combinatorial invariant in the common sense of the word. 


\subsubsection{Definition.}

The sheaf of ideals $\mathcal{A}(\delta) \subset \mathcal{O}_{\mathbf{P}^{2}}$ such that $\operatorname{Supp}\left(\mathcal{O}_{\mathbf{P}^{2}} / \mathcal{A}\right) \subset \operatorname{Sing} \mathcal{C}$ is called the sheaf of ideals of quasiadjunction corresponding to the face of quasiadjunction $\delta$ if the stalk $\mathcal{A}_{p}$ at each singular point $p \in \mathcal{C}$ with local ring $\mathcal{O}_{p}$ is the ideal $A$ of quasiadjunction corresponding to the face $\Delta_{p}=\Delta \cap H_{p}$ with $H_{p} \subset \mathbf{R}^{r}$ being given by $x_{i_{j}}=0$ where $i_{j}$ are the coordinates corresponding to the components of $\mathcal{C}$ not passing through $p$.

\subsubsection{Examples.}

1.(cf. [L2]) For an irreducible curve of degree $d$ with nodes and the ordinary cusps as the only singularities the global polytope of quasiadjunction coincide with the local one of the cusp. The only face of quasiadjunction is $x=\frac{1}{6}$. The contributing hyperplane is given by $d x=\frac{d}{6}$ and its level is $\frac{d}{6}$. The sheaf of quasiadjunction corresponding to this face of quasiadjunction is the ideal sheaf having stalks different from the local ring only at the points of $\mathbf{P}^{2}$ where the curve has cusps and the stalks at those points are the maximal ideals of the corresponding local rings.

2. Let us consider $\mathcal{C}$ which is an arrangement of lines. For a point $P$ let $m_{P}$ denotes the multiplicity. We consider only points with $m_{P}>2$. Each global face of quasiadjunciton is a solution of a system of equations:

$$
L_{P}: \quad x_{i_{1}}+\ldots+x_{i_{m}}=s_{P}
$$

where $s_{P}=1, \ldots, m_{P}-2$ (cf. example 3 in 2.5$)$. The indices of variables $x$ correspond to the lines of the arrangement and $x_{i}$ appears in $L_{P}$ if and only if it correspond to a line passing through $P$. Each system (2.6.1) corresponding to a face of quasiadjunciton singles out a collection of vertices of the arrangement. This face is contributing if the equation

$$
x_{1}+\ldots+x_{r}=k, \quad(k \in \mathbf{N})
$$

is a linear combination of equations (2.6.1). The level of such contributing face is $k$. Its order is the g.c.d of minors of maximal order in system (2.6.1). 


\section{The first Betti number of an abelian cover.}

In this section we shall prove a formula for the irregularity of an abelian covers of $\mathbf{P}^{2}$ branched over $\mathcal{C}$ in terms of the polytopes of quasiadjunction introduced in the last section. More precisely we shall calculate the multiplicity of a character of the Galois group of the cover acting on the space $H^{1,0}\left(\tilde{V}_{m_{1}, . ., m_{r}}\right)$ of holomorphic 1-forms. We translate this into an information about characteristic varieties of the fundamental group and consider several examples of characteristic varieties for the fundamental groups of the complements to arrangements of lines.

\subsection{Statement of the theorem.}

Let $\mathcal{C}=\cup_{i=1}^{i=r} C_{i}$ be a reduced curve $f(u, x, y)=f_{1}(u, x, y) \cdots f_{r}(u, x, y)$ with $r$ irreducible components and the degrees of components equal to $d_{1}, . ., d_{r}$ and $d=d_{1}+\ldots+d_{r}$ be the total degree of $f(u, x, y)=0$. Let $L_{\infty}$ be the line $u=0$ at infinity which, as above, we shall assume transversal to $\mathcal{C}$ (cf.1.1).

a) The irregularity of a desingularization $\widetilde{V}_{m_{1}, \ldots, m_{r}}$ of an abelian cover of $\mathbf{P}^{2}$ branched over $\mathcal{C} \cup L_{\infty}$ and corresponding to the surjection $\pi_{1}\left(\mathbf{P}^{2}-\mathcal{C} \cup\right.$ $\left.L_{\infty}\right) \rightarrow \mathbf{Z} / m_{1} \mathbf{Z} \oplus \ldots \oplus \mathbf{Z} / m_{r} \mathbf{Z}$ is equal to

$$
\Sigma_{\mathcal{C}^{\prime}}\left(\Sigma_{\delta\left(\mathcal{C}^{\prime}\right)} N\left(\delta\left(\mathcal{C}^{\prime}\right)\right) \cdot \operatorname{dim} H^{1}\left(\mathcal{A}_{\delta\left(\mathcal{C}^{\prime}\right)}(d-3-l(\delta(\mathcal{C})))\right)\right)
$$

where the summations are over all curves $\mathcal{C}^{\prime}$ formed by the components of $\mathcal{C}$ and the contributing faces of quasiadjunction $\delta\left(\mathcal{C}^{\prime}\right)$ respectively. Here $l\left(\delta\left(\mathcal{C}^{\prime}\right)\right)$ is the level of the contributing face of $\delta\left(\mathcal{C}^{\prime}\right)$ and $N\left(\delta\left(\mathcal{C}^{\prime}\right)\right)$ is the number of interior $\mathcal{C}^{\prime}$-points $\left(\frac{i_{1}+1}{m_{1}}, \ldots, \frac{i_{r}+1}{m_{r}}\right)$ in the contributing face of $\delta\left(\mathcal{C}^{\prime}\right)$.

b) Let $\chi_{j}$ be the character of $\mathbf{Z}_{m_{1}} \oplus \ldots \oplus \mathbf{Z}_{m_{r}}$ taking on $\left(a_{1}, . ., a_{j}, . ., a_{r}\right)$ value $\exp \left(2 \pi \sqrt{-1} \frac{a_{j}}{m_{j}}\right)$. For a character $\chi$ of $\mathbf{Z}_{m_{1}} \oplus \ldots \oplus \mathbf{Z}_{m_{r}}$ let

$$
H_{\chi}^{1,0}\left(\widetilde{V}_{m_{1}, \ldots, m_{r}}\right)=\left\{x \in H^{1,0}\left(\widetilde{V}_{m_{1}, \ldots, m_{r}}\right), g \in \mathbf{Z}_{m_{1}} \oplus \ldots \oplus \mathbf{Z}_{m_{r}} \mid g \cdot x=\chi(g) \cdot x\right\} .
$$

If $\left(\frac{i_{1}+1}{m_{1}}, \ldots, \frac{i_{r}+1}{m_{r}}\right)$ is an interior $\mathcal{C}$-point (cf. 2.3.2) belonging to the contributing face $\delta$ then

$$
\operatorname{dim} H_{\chi_{1}^{i_{1} \ldots \chi_{r}}}^{1}\left(\widetilde{V}_{m_{1}, \ldots, m_{r}}\right)=\operatorname{dim} H^{1}\left(\mathcal{A}_{\delta}(d-3-l(\delta))\right)
$$


c) Let $t_{i}=\exp \left(2 \pi \sqrt{-1} x_{i}\right)$. For each contributing face $\delta$ belonging to $\mathcal{U}^{\circ}$ and its image $\bar{\delta}$ under the conjugation map (cf. 2.3), let $L_{s}\left(x_{1}, . ., x_{r}\right)=\beta_{s}$ be the system of equations defining it where $L_{s}\left(x_{1}, \ldots x_{r}\right)$ is a linear form with integer coefficients such that g.c.d. of the minors of maximal order in the matrix of coefficients is equal to 1 . Then the corresponding essential component of the characteristic variety of $\pi_{1}\left(\mathbf{P}^{2}-\mathcal{C} \cup L_{\infty}\right)$, which either has a positive dimension or is a torsion point, is the intersection of cosets given by the equations:

$$
\exp \left(2 \pi \sqrt{-1} L_{s}\right)=\exp \left(2 \pi \sqrt{-1} \beta_{s}\right)
$$

written in terms of $t_{i}$ 's. Vice versa, any essential component can be obtained in such way.

Note that c) implies that the essential components of the characteristic varieties are Zariski's closures of the images of the contributing faces under the exponential map. Indeed, since g.c.d. of minors of coefficients in $L_{s}$ is 1 the intersection of subgroups $\exp \left(2 \pi \sqrt{-1} L_{s}\right)=1$ is connected and the closure of the image of the face of quasiadjunction is Zariski dense in the translation of this connected component given by (3.1.3).

\subsection{Proof of the Theorem}

We shall start with the case when $m_{i} \geq d_{i}$ for $i=1, \ldots, r$. Let $\mathcal{A} \subset \mathcal{O}_{\mathbf{P}^{r+2}}$ be the sheaf of adjoint ideals of the complete intersection $V_{m_{1}, \ldots, m_{r}} \subset \mathbf{P}^{r+2}$ given by the equations (cf. (1.3.1.1)):

$$
z_{1}^{m_{1}}=u^{m_{1}-d_{1}} f_{1}(u, x, y), \ldots, z_{r}^{m_{r}}=u^{m_{r}-d_{r}} f_{r}(u, x, y)
$$

$V_{m_{1}, \ldots, m_{r}}$ provides a model of an abelian branched cover of $\mathbf{P}^{2}$ branched over $f_{1} \cdots f_{r}=0$ and the line at infinity. $V_{m_{1}, \ldots, m_{r}}$ has isolated singularities at the points of (3.2.1) which are above the singularities of $\mathcal{C}$ in $\mathbf{P}^{2}-L_{\infty}$. The action of the Galois group of the cover is induced from the action of the product of groups of roots of unity $\mu_{m_{1}} \times \cdots \times \mu_{m_{r}}$ on the $\mathbf{P}^{r+2}$ via multiplication of corresponding $z$-coordinates.

Let $H$ be the set of common zeros of $z_{1}, \ldots, z_{r} \in H^{0}\left(\mathbf{P}^{r+2}, \mathcal{O}(1)\right)$ and $\mathcal{A}_{i_{1}, \ldots, i_{r}}$ be the subsheaf of $\mathcal{O}_{\mathbf{P}^{r+2}}$ germs of section product of which with $z_{1}^{i_{1}} \cdots z_{r}^{i_{r}}$ belongs to $\mathcal{A}$. The action of $\mu_{m_{1}} \times \cdots \times \mu_{m_{r}}$ on $\mathbf{P}^{r+2}$ induces the action on $\mathcal{A}_{i_{1}, \ldots, i_{r}}$. 
Let $\mathcal{J}_{H}$ be the ideal sheaf of the plane $H \subset \mathbf{P}^{2}$. We have the following $\mu_{m_{1}} \times \cdots \times \mu_{m_{r}}$-equivariant sequence:

$$
\begin{gathered}
0 \rightarrow \mathcal{A}_{i_{1}, \ldots, i_{r}}\left(\left(m_{1}-i_{1}\right)+\ldots+\left(m_{r}-i_{r}\right)-r-3\right) \otimes \mathcal{J}_{H} \rightarrow \mathcal{A}_{i_{1}, \ldots, i_{r}}\left(\left(m_{1}-i_{1}\right)+\ldots+\left(m_{r}-i_{r}\right)-r-3\right) \\
\left.\rightarrow \mathcal{A}_{i_{1}, . ., i_{r}}\left(\left(m_{1}-i_{1}\right)+\ldots\left(m_{r}-i_{r}\right)-r-3\right)\right|_{H} \rightarrow 0
\end{gathered}
$$

Let

$$
\begin{gathered}
F\left(i_{1}, \ldots, i_{r}\right)=\operatorname{dim} H^{1}\left(\mathcal{A}_{i_{1}, \ldots, i_{r}}\left(\left(m_{1}-i_{1}\right)+\ldots+\left(m_{r}-i_{r}\right)-r-3\right)\right) \\
F_{\chi}\left(i_{1}, \ldots, i_{r}\right)=\operatorname{dim}\left\{x \in H ^ { 1 } \left(\mathcal{A}_{i_{1}, \ldots, i_{r}}\left(\left(m_{1}-i_{1}\right)+\ldots+\left(m_{r}-i_{r}\right)-r-3\right) \mid g \cdot x=\chi(g) x,\right.\right. \\
\left.\forall g \in \mu_{m_{1}} \times \cdots \mu_{m_{r}}\right\}
\end{gathered}
$$

In particular $F(0, \ldots, 0)$ is the irregularity of a nonsingular model of $V_{m_{1}, \ldots, m_{r}}$.

Step 1. Degree of the curves in the linear system $H^{0}\left(\mathcal{A}_{i_{1}, \ldots, i_{r}}\left(\left(m_{1}-i_{1}\right)+\right.\right.$ $\left.\left.\ldots+\left(m_{r}-i_{r}\right)-r-3\right)\left.\right|_{H}\right)$. Let us calculate the multiplicity of the line $L_{\infty}: z_{1}=$ $\ldots=z_{r}=u=0$ as the fixed component of the curves in the linear system cut on $H$ by the hypersurfaces in the linear system $H^{0}\left(\mathcal{A}_{i_{1}, \ldots, i_{r}}\left(\left(m_{1}-i_{1}\right)+\ldots+\right.\right.$ $\left.\left.\left(m_{r}-i_{r}\right)-r-3\right)\right)$. This multiplicity is the smallest $k$ such that $u^{k}$ belongs to the latter system of hypersurfaces. In appropriate coordinates $\left(z_{1}, \ldots, z_{r}, u, v\right)$ at a point $P$ of this line outside of $L_{\infty} \cap \mathcal{C}$ (i.e. we have $f_{1}(P) \cdots f_{r}(P) \neq 0$ ) the local equation of $V_{m_{1}, \ldots, m_{r}}$ is $z_{1}^{m_{1}}=u^{m_{1}-d_{1}}, \ldots, z_{r}^{m_{r}}=u^{m_{r}-d_{r}}$. Let

$$
\begin{gathered}
l=l . c . m .\left(\frac{m_{1}}{m_{1}-d_{1}}\left(m_{1}-d_{1}\right) \cdots\left(m_{r}-d_{r}\right), \ldots,\right. \\
\left.\ldots \frac{m_{r}}{m_{r}-d_{r}}\left(m_{1}-d_{1}\right) \cdots\left(m_{r}-d_{r}\right),\left(m_{1}-d_{1}\right) \cdots\left(m_{r}-d_{r}\right)\right)
\end{gathered}
$$

Then each branch of the normalization of $V_{m_{1}, \ldots, m_{r}}$ has the parameterization $(t, v)$ such that:

$$
z_{1}=t^{\frac{l\left(m_{1}-d_{1}\right)}{m_{1}\left(m_{1}-d_{1}\right) \cdots\left(m_{r}-d_{1}\right)}}, \ldots, z_{r}=t^{\frac{l\left(m_{r}-d_{r}\right)}{m_{r}\left(m_{1}-d_{1}\right) \cdots\left(m_{r}-d_{r}\right)}}, u=t^{\frac{l}{\left(m_{1}-d_{1}\right) \cdots\left(m_{r}-d_{r}\right)}}
$$

Therefore the pull back of the form $\left.\frac{z_{1}^{i_{1} \ldots z_{r}^{i_{r}} u^{k} d u \wedge d v}}{z_{1}^{m_{1}-1} \ldots z_{r}^{m_{r}-1}}\right|_{V_{m_{1}, \ldots, m_{r}}}$ to the $(t, v)$ chart is regular if and only if

$$
k>\Sigma_{j=1}^{j=r}\left(m_{j}-d_{j}-\left(i_{j}+1\right)\right)+\frac{d_{j}\left(i_{j}+1\right)}{m_{j}}-1
$$


The smallest $k$ which satisfies this inequality, i.e. the multiplicity of the line $u=0$ as the component of a generic curve from $H^{0}\left(\mathcal{A}_{i_{1}, \ldots, i_{r}}\left(\left(m_{1}-i_{1}\right)+\right.\right.$ $\left.\left.\ldots\left(m_{r}-i_{r}\right)-r-3\right)\left.\right|_{H}\right)$, is equal to

$$
\Sigma_{j}\left(m_{j}-d_{j}-\left(i_{j}+1\right)\right)+\left[\Sigma_{j} \frac{d_{j}\left(i_{j}+1\right)}{m_{j}}\right]
$$

A consequence of this is that the degree of the moving curves in the linear system $H^{0}\left(\mathcal{A}_{i_{1}, \ldots, i_{r}}\left(\left(m_{1}-i_{1}\right)+\ldots\left(m_{r}-i_{r}\right)-r-3\right) \mid H\right)$ is equal to $\Sigma_{j} d_{j}-$ $3-\left[\Sigma_{j} \frac{d_{j}\left(i_{j}+1\right)}{m_{j}}\right]$ and therefore the moving curves belong to the linear system $H^{0}\left(\mathcal{A}_{\Delta}\left(\left(\Sigma_{j} d_{j}\right)-3-\left[\frac{d_{j}\left(i_{j}+1\right)}{m_{j}}\right]\right)\right.$ where $\Delta$ is the polytope of quasiadjunction containing $\left(\frac{i_{1}+1}{m_{1}}, \ldots, \frac{i_{r}+1}{m_{r}}\right)$. In fact the moving curves form a complete system since the cone over any curve in $H^{0}\left(\mathcal{A}_{\Delta}\left(\Sigma_{j} d_{j}-3-\left[\Sigma_{j} \frac{d_{j}\left(i_{j}+1\right)}{m_{j}}\right]\right)\right.$ belongs to $H^{0}\left(\mathcal{A}_{i_{1}, \ldots, i_{r}}\left(\Sigma_{j}\left(m_{j}-i_{j}\right)-r-3\right)\right)$.

Step 2. A recurrence relation for $F\left(i_{1}, \ldots, i_{r}\right)$ and $F_{\chi}\left(i_{1}, \ldots, i_{r}\right)$. Let $s\left(i_{1}, \ldots, i_{r}\right)=$ $\operatorname{dim} H^{1}\left(\mathcal{A}_{\Delta}\left(\Sigma_{j} d_{j}-3-\left[\Sigma_{j} \frac{d_{j}\left(i_{j}+1\right)}{m_{j}}\right]\right)\right.$ where $\Delta$ is the polytope of quasiadjunction of $\mathcal{C}$ containing $\left(\frac{i_{1}+1}{m_{1}}, \ldots, \frac{i_{r}+1}{m_{r}}\right)$ and $\epsilon_{\chi}\left(i_{1}, \ldots, i_{r}\right)=1$ (resp. 0$)$ if $\chi=\chi_{1}^{i_{1}-m_{1}+1} \cdots \chi_{r}^{i_{r}-m_{r}+1}$ (resp. otherwise). We claim the following recurrence:

$$
\begin{gathered}
F\left(i_{1}, \ldots, i_{r}\right)=s\left(i_{1}, \ldots, i_{r}\right)+\Sigma_{l=1}^{l=r}(-1)^{l+1} \Sigma_{i_{j_{1}}<\ldots<i_{j_{l}}} F\left(\ldots, i_{j_{1}}+1, \ldots, i_{j_{l}}+1, \ldots\right) ; \\
F_{\chi}\left(i_{1}, \ldots, i_{r}\right)=\epsilon_{\chi}\left(i_{1}, \ldots, i_{r}\right) s\left(i_{1}, \ldots, i_{r}\right)+ \\
\sum_{l=1}^{l=r}(-1)^{l+1} \Sigma_{i_{j_{1}}<\ldots<i_{j_{l}}} F_{\chi\left(\chi_{j_{1}} \cdots \chi_{j_{l}}\right)^{-1}\left(\ldots, i_{j_{1}}+1, \ldots, i_{j_{l}}+1, \ldots\right)}
\end{gathered}
$$

Equivalently the first of equalities (3.2.6) can be written as

$$
s\left(i_{1}, \ldots, i_{r}\right)=\Sigma_{l=0}^{l=r}(-1)^{l} \Sigma_{i_{j_{1}}<\ldots<i_{j_{l}}} F\left(\ldots, i_{j_{1}}+1, \ldots, i_{j_{l}}+1, \ldots\right)
$$

and similarly for the second. This identity will be derived from the following. For $h$ such that $1 \leq h \leq r$ let

$F\left(i_{1}, . ., i_{r} \mid q_{1}, \ldots, q_{h}\right)=\operatorname{dim} H^{1}\left(\left.\mathcal{A}_{i_{1}, . ., i_{r}}\left(\left(m_{1}-i_{1}\right)+\ldots\left(m_{r}-i_{r}\right)-r-3\right)\right|_{H_{q_{1}} \cap \ldots H_{q_{h}}}\right)$

where $H_{s}$ is the hyperplane $z_{s}=0$ in $\mathbf{P}^{r+2}$ while for $h=0$ we let $F\left(i_{1}, \ldots, i_{r} \mid \emptyset\right)=$ $F\left(i_{1}, \ldots, i_{r}\right)$. In particular $s\left(i_{1}, . ., i_{r}\right)=F\left(i_{1}, \ldots i_{r} \mid 1, \ldots, r\right)$. 
Similarly one defines $F_{\chi}\left(i_{1}, \ldots, i_{r} \mid q_{1}, \ldots, q_{h}\right)$. We shall prove by induction over $h$ :

$$
\begin{gathered}
F\left(i_{1}, \ldots, i_{r} \mid q_{1}, \ldots, q_{h}\right)= \\
\sum_{l=0}^{l=h}(-1)^{l} \sum_{i_{j_{1}}<\ldots<i_{j_{l}}, i_{j_{1}}, \ldots, i_{j_{l}} \subset\left(q_{1}, \ldots, q_{h}\right)} F\left(\ldots, i_{j_{1}}, \ldots, i_{j_{l}}, \ldots\right) \\
F_{\chi}\left(i_{1}, \ldots, i_{r} \mid q_{1}, \ldots, q_{h}\right)= \\
\sum_{l=0}^{l=h}(-1)^{l} \sum_{i_{j_{1}}<\ldots<i_{j_{l}},\left(i_{j_{1}}, \ldots, i_{j_{l}}\right) \subset\left(q_{1}, \ldots, q_{h}\right)} F_{\chi \cdot \chi_{j_{1}}^{-1} \ldots \chi_{j_{l}}^{-1}}\left(\ldots, i_{j_{1}}+1, \ldots, i_{j_{l}}+1, \ldots\right)
\end{gathered}
$$

The identity (3.2.6) is a special case of (3.2.7) when $q_{i}=i$. For any $\left(i_{1}, \ldots, i_{r} \mid q_{1}, \ldots, q_{h}\right),(h \geq 0)$ from the exact sequence (in which the left map is the multiplication by $\left.z_{q_{h+1}}\right)$ :

$$
\begin{gathered}
\left.0 \rightarrow \mathcal{A}_{\ldots, i_{q_{h+1}+1}, \ldots}\left(\ldots+\left(m_{q_{h+1}}-i_{q_{h+1}}-1\right)+\ldots-r-3\right)\right|_{H_{q_{1}} \cap \ldots \cap H_{q_{h}}} \rightarrow \\
\left.\mathcal{A}_{i_{1}, . ., i_{r}}\left(\left(m_{1}-i_{1}\right)+\ldots+\left(m_{r}-i_{r}\right)-r-3\right)\right|_{H_{q_{1}} \cap \ldots \cap H_{q_{h}}} \rightarrow \\
\left.\mathcal{A}_{i_{1}, . ., i_{r}}\left(\left(m_{1}-i_{1}\right)+\ldots+\left(m_{r}-i_{r}\right)-r-3\right)\right|_{H_{q_{1}, \ldots, q_{h}, q_{h+1}}} \rightarrow 0
\end{gathered}
$$

we obtain

$$
\begin{gathered}
F\left(i_{1}, \ldots, i_{r} \mid q_{1}, \ldots, q_{h}, q_{h+1}\right)=-F\left(i_{1}, \ldots, i_{q_{h+1}}+1, \ldots i_{r} \mid q_{1}, . ., q_{h}\right)+F\left(i_{1}, \ldots, i_{r} \mid q_{1}, . ., q_{h}\right) \\
F_{\chi}\left(i_{1}, \ldots, i_{r} \mid q_{1}, \ldots, q_{h}, q_{h+1}\right)=-F_{\chi \cdot \chi_{q_{h+1}}^{-1}}\left(i_{1}, \ldots, i_{q_{h+1}+1}, \ldots, i_{r} \mid q_{1}, \ldots, q_{h}\right)+ \\
F_{\chi}\left(i_{1}, \ldots, i_{r} \mid q_{1}, . ., q_{h}\right) .
\end{gathered}
$$

Indeed, the map

$$
\begin{array}{r}
\left.H^{0}\left(\mathcal{A}_{i_{1}, . ., i_{r}}\left(\left(m_{1}-i_{1}\right)+\ldots+\left(m_{r}-i_{r}\right)\right)-r-3\right)\right|_{H_{q_{1}, \ldots, q_{h}}} \rightarrow \\
\left.\rightarrow H^{0}\left(\mathcal{A}_{i_{1}, . ., i_{r}}\left(\left(m_{1}-i_{1}\right)+\ldots+\left(m_{r}-i_{r}\right)\right)-r-3\right)\right|_{H_{q_{1}, \ldots, q_{h}, q_{h+1}}}
\end{array}
$$

is surjective because the cone in $H_{q_{1}} \cap \ldots \cap H_{q_{h}}$ over the hypersurface in $H^{0}\left(\left.\mathcal{A}_{i_{1}, . ., i_{r}}\left(\left(m_{1}-i_{1}\right)+\ldots+\left(m_{r}-i_{r}\right)-r-3\right)\right|_{H_{q_{1}, \ldots, q_{h}, q_{h+1}}}\right)$ belongs to $H^{0}\left(\mathcal{A}_{i_{1}, . ., i_{r}}\left(\left(m_{1}-\right.\right.\right.$ $\left.\left.\left.i_{1}\right)+\ldots+\left(m_{r}-i_{r}\right)-r-3\right)\left.\right|_{H_{q_{1}, \ldots, q_{h}}}\right)$. Moreover for $q_{i}=i, i=1, \ldots, r$ we have $F_{\chi}\left(i_{1}, \ldots, i_{r} \mid 1, \ldots r\right)=\epsilon_{\chi}\left(i_{1}, \ldots, i_{r}\right) s\left(i_{1}, \ldots, i_{r}\right)$ since to $\phi(x, y) \in H^{0}\left(\mathcal{A}_{i_{1}, \ldots, i_{r}}\right)$ corresponds the form $\psi=z_{1}^{m_{1}-i_{1}-1} \cdots z_{r}^{m_{r}-i_{r}-1} \pi^{*} \phi$ holomorphic on $\widetilde{V}_{m_{1}, \ldots, m_{r}}$ and satisfying: $g^{*}(\psi)=\chi_{1}^{i_{1}-m_{1}+1} \cdots \chi_{r}^{i_{r}-m_{r}+1} \psi$. This shows that $(3.2 .7)$ is valid for $h=1$ and that validity of $(3.2 .7)$ for the array $\left(q_{1}, . ., q_{h+1}\right)$ provided it is valid for all $\left(q_{1}, \ldots, q_{h}\right)$. 
Step 3. An explicit formula for $F\left(i_{1}, \ldots, i_{r}\right)$. Let $\mathcal{C}\left(j_{1}, \ldots, j_{s}\right)=C_{j_{1}} \cup \ldots \cup C_{j_{s}}$ be a curve formed by a union of the components of $\mathcal{C}$ and let

$F_{\mathcal{C}\left(j_{1}, \ldots, j_{s}\right)}\left(i_{1}, \ldots, i_{s}\right)=\operatorname{dim} H^{1}\left(\mathcal{A}\left(\mathcal{C}\left(j_{1}, \ldots, j_{s}\right)\right)_{i_{1}, \ldots, i_{s}}\left(\left(m_{j_{1}}-i_{1}\right)+\ldots+\left(m_{j_{s}}-i_{s}\right)+s-3\right)\right.$.

Note that

$$
i_{j}=m_{j}-1\left(j \neq j_{1}, \ldots j_{s}\right) \Rightarrow F\left(i_{1}, \ldots, i_{r}\right)=F_{\mathcal{C}\left(j_{1}, \ldots, j_{s}\right)}\left(i_{1}, \ldots, i_{s}\right)
$$

since the local conditions defining both sheaves coincide (indeed:

$$
\left.\frac{z_{1}^{i_{1}} \cdots z_{j_{k}}^{m_{j_{k}}-1} \cdots z_{r}^{i_{r}} d x \wedge d y}{z_{1}^{m_{1}-1} \ldots z_{r}^{m_{r}-1}}=\frac{z_{1}^{i_{1}} \cdots z_{i_{k}}^{m_{i_{k}}-1} \cdots z_{r}^{i_{r}} d x \wedge d y}{z_{1}^{m_{1}-1} \cdots z_{i_{j_{k}}}^{m_{j_{k}}-1} \cdots z_{r}^{m_{r}-1}}\right)
$$

as well as the degrees of the curves in the corresponding linear systems. Moreover

$$
F_{\mathcal{C}\left(j_{1}, \ldots, j_{s}\right)}(0, \ldots, 0)
$$

is the irregularity of the cover of $\mathbf{P}^{2}$ branched over $\mathcal{C}\left(j_{1}, \ldots, j_{s}\right)$ and having the ramification index $m_{i}$ over the component $C_{i}\left(i=j_{1}, \ldots, j_{s}\right)$.

We solve the recurrence relation (3.2.6) subject to the "initial condition" (3.2.11). It is convenient to view each relation (3.2.6) as the one connecting the values of the function defined at the vertices of the integer lattice in the parallelepiped $0 \leq x_{i} \leq m_{i},(i=1, \ldots, r)$. Each equation connects the values of this function at the vertices of a parallelepiped with sides equal to 1 . It is clear that the sum of all equations (3.2.6) yields:

$$
\begin{gathered}
F(0, \ldots, 0)=\Sigma_{0 \leq i_{s}<m_{s}-1} s\left(i_{1}, \ldots, i_{r}\right)+\Sigma_{l=1}^{l=r-1} \Sigma_{\left(j_{1}<\ldots<j_{l}\right)} F_{\mathcal{C}\left(j_{1}, \ldots, j_{l}\right)}(0, \ldots 0) \\
F_{\chi}(0, \ldots, 0)=\Sigma_{0 \leq i_{s}<m_{s}-1} \epsilon_{\chi}\left(i_{1}, . .,, i_{r}\right) s\left(i_{1}, \ldots, i_{r}\right)+\Sigma_{l=1}^{l=r-1} \Sigma_{\left(j_{1}<\ldots<j_{l}\right)} F_{\mathcal{C}\left(j_{1}, \ldots, j_{l}\right)_{\chi}}(0, \ldots, 0)
\end{gathered}
$$

Remark. Alternative derivation of (3.2.12).

Sheaves $\mathcal{A}_{\Delta}\left(d_{1}+\ldots+d_{r}-r-2-\left[\Sigma_{j} \frac{d_{j}\left(i_{j}+1\right)}{m_{j}}\right]\right)$ admit the following interpretation also yielding (3.2.12). Let us consider the following global version of the diagram (2.3.1):

$$
\begin{array}{ccc}
\bar{V}_{m_{1}, \ldots, m_{r}} & \bar{\pi} & Y_{\mathcal{C}} \\
\downarrow \bar{\rho} & \rightarrow & \downarrow \rho \\
V_{m_{1}, \ldots, m_{r}} & \pi & \mathbf{P}^{2}
\end{array}
$$


Here $\rho: Y_{\mathcal{C}} \rightarrow \mathbf{P}^{2}$ is an embedded resolution of singularities of $\mathcal{C}$ which are worse than nodes, $\bar{V}_{m_{1}, \ldots, m_{r}}$ is the normalization of $V_{m_{1}, \ldots, m_{r}} \times{ }_{\mathbf{P}^{2}} Y_{\mathcal{C}}$ and $\bar{\pi}, \bar{\rho}$ are the obvious projections. Let

$$
\bar{\pi}_{*}\left(\mathcal{O}_{\bar{V}_{m_{1}, \ldots, m_{r}}}\right)=\oplus \mathcal{L}_{\chi_{1}^{i_{1} \ldots \chi_{r}}}^{-1}
$$

be the decomposition by the characters of the Galois group acting on $\bar{\pi}_{*}\left(\mathcal{O}_{\bar{V}_{m_{1}}, \ldots, m_{r}}\right)$. Then we have:

$\mathcal{A}_{\Delta}\left(\Sigma_{j} d_{j}-3-\left[\Sigma_{j} \frac{d_{j}\left(i_{j}+1\right)}{m_{j}}\right]\right)=\rho_{*}\left(\bar{\pi}_{*}\left(\Omega_{\bar{V}_{m_{1}, \ldots, m_{r}}}\right) \otimes \mathcal{L}_{\chi\left(m_{1}-\left(i_{1}+1\right), \ldots, m_{r}-\left(i_{r}+1\right)\right)}\right)$

where $\Delta$ is the polytope of quasiadjunction containing $\left(\frac{i_{1}+1}{m_{1}}, \ldots, \frac{i_{r}+1}{m_{r}}\right)$. Indeed it follows from (2.3.8) that a germ $\phi$ of a holomorphic function belongs to the sheaf in the left side of (3.2.15) if and only if the order of $\phi$ along an exceptional curve $E_{k} \subset Y_{\mathcal{C}}$ satisfies: $\operatorname{ord}_{E_{k}} \phi \geq \sum a_{k, j}\left(\frac{m_{j}-\left(i_{j}+1\right)}{m_{j}}-c_{k}\right)$ and the sheaf on the left is a subsheaf of $\mathcal{O}_{\mathbf{P}^{2}}\left(\left[\sum_{j} d_{j}\left(\frac{m_{j}-\left(i_{j}+1\right)}{m_{j}}\right)\right]\right)$ with the quotient having a zero-dimensional support. One readily sees that the sheaf on the right has the same local description. This identity also implies (3.2.12) as follows from Serre's duality and (3.2.14).

Step 4. A vanishing result.

If $\Delta$ is a polytope of quasiadjunction, $\Xi_{k}=\left\{\left(x_{1}, \ldots, x_{r}\right) \in \mathcal{U} \mid k \leq d_{1} x_{1}+\right.$ $\left.\ldots+d_{r} x_{r}<k+1\right\}$ and $k$ is such that $\Delta \cap \Xi_{k} \neq \emptyset$ then

$$
H^{1}\left(\mathcal{A}_{\Delta}(d-r-2-k)\right)=0
$$

unless $\Delta$ is a contributing polytope of quasiadjunction and $\Delta \cap \Xi_{k}$ is a face of quasiadjunction.

If $\Delta$ isn't contributing (cf. 2.6.2), then the intersection $\Delta \cap \Xi_{k}$ has a positive volume. If $X(n)$ is the number of points $\left(\frac{i}{n}, \ldots, \frac{i}{n}\right)$ in the latter, it follows from (3.2.12) that we have $b_{1}(\mathcal{C}, n) \geq \operatorname{dim}^{1}\left(\mathcal{A}_{\Delta}(d-r-2-k)\right) \cdot X(n)$. We have $X(n)>C \cdot n^{r}$ for some non zero constant $C$. Therefore we get contradiction with Corollary (1.3.3) unless $\operatorname{dim} H^{1}\left(\mathcal{A}_{\Delta}(d-r-2-k)\right)=0$.

Step 5. End of the proof. Step 4 and the formula (3.2.12) give a) and b) of the theorem in the case $m_{i} \geq d_{i}$ for $i=1, . ., i_{r}$.

If $\chi$ is a character of $\mathbf{Z}_{m_{1}} \oplus \ldots \oplus \mathbf{Z}_{m_{r}}$ acting on $H^{0}\left(\Omega_{\tilde{V}_{m_{1}, \ldots, m_{r}}}^{1}\right)=H^{1,0}\left(\tilde{V}_{m_{1}, \ldots, m_{r}}\right)$ then $\bar{\chi}$ is a character with eigenspace of the same dimension for the action 
of $\mathbf{Z}_{m_{1}} \oplus \ldots \oplus \mathbf{Z}_{m_{r}}$ on $H^{0,1}\left(\tilde{V}_{m_{1}, ., m_{r}}\right)$. Hence part b) and Sakuma formula (cf. 1.3.2) imply that a points $\left(\ldots \frac{i_{j}+1}{m_{j}} \ldots\right)$ belongs to a contributing face of $\Delta$ or its conjugate if and only if $\left(\ldots, \exp \left(2 \pi \sqrt{-1} \frac{i_{j}+1}{m_{j}}\right), \ldots\right)$ belongs to $i$-th characteristic variety with $i=\operatorname{dim} H^{1}\left(\mathcal{A}_{\Delta}\left(d_{1}+\ldots+d_{r}-r-3-k(\Delta)\right)\right.$. Since a characteristic variety is a translated by a point of finite order subtorus (cf. 1.4.2) this implies c). Now the remaining cases of the formula a) follows from Sakuma's result (1.3.2.2).

\subsection{Examples.}

In 2.6.4 we did describe systems of equations for faces of quasiadjunction in the case of arrangements of lines. To determine if a set of solutions of the system corresponding to a face $\delta$ actually corresponds to a component of characteristic variety one should

a) calculate the superabundance (3.1.2) of the corresponding linear system and

b) decide the "amount of translation" i.e. to normalize the system of equations so that the g.c.d. of minors of the left hand sides of (2.6.1) will be equal to one.

In any event, if superabundance is not zero, then clearly the component of characteristic variety will be a connected component of the subgroup given by the equations: $\exp \left(L_{P}\right)=1$ with $P$ running through all vertices singled out by the face of quasiadjunction.

Example 1. Let us calculate the irregularity of the abelian cover of $\mathbf{P}^{2}$ branched over the arrangement $L: u v(u-v) w=0$ and corresponding to the homomorphism $H_{1}\left(\mathbf{P}^{2}-L\right)=\mathbf{Z}^{3} \rightarrow(\mathbf{Z} / n \mathbf{Z})^{3}$. The only nontrivial ideal of quasiadjunction is the maximal ideal of the local ring with corresponding polytope of quasiadjunction: $x+y+z>1$. Hence the irregularity of the abelian cover is $\operatorname{Card}\left\{(i, j) \mid 0<i<n, 0<j<n, \frac{i}{n}+\frac{j}{n}+\frac{k}{n}=\right.$ $1\} \cdot \operatorname{dim} H^{1}(\mathcal{J}(3-3-1))$ where $\mathcal{J}=\operatorname{Ker} \mathcal{O} \rightarrow \mathcal{O}_{P}$ where $P: u=v=0 . \mathcal{J}$ has the following Koszul resolution:

$$
0 \rightarrow \mathcal{O}(-2) \rightarrow \mathcal{O}(-1) \oplus \mathcal{O}(-1) \rightarrow \mathcal{J} \rightarrow 0
$$

which yields $H^{1}(\mathcal{J}(-1))=H^{2}(\mathcal{O}(-3))=$ C. Now the counting points on $x+y+z=1$ yields $\frac{n^{2}-3 n+2}{2}$ as the irregularity of the abelian cover. 
Example 2. Let us consider the arrangement formed by the sides of an equilateral triangle $\left(x_{1}, x_{2}, x_{3}\right)$ and its medians $\left(x_{4}, x_{5}, x_{6}\right)$ arranged so that the vertices are the intersection points of $\left(x_{1}, x_{2}, x_{4}\right),\left(x_{2}, x_{3}, x_{5}\right)$ and $x_{3}, x_{1}, x_{6}$ respectively (Ceva arrangement cf. [BHH]). It has 6 lines, 4 triple and 3 double points. The polytopes of quasiadjunction are the connected components of the partition of $\mathcal{U}=\left\{\left(x_{1}, . ., x_{6}\right) \mid 0 \leq x_{i} \leq 1, i=1, \ldots 6\right\}$ by the hyperplanes:

$$
x_{1}+x_{2}+x_{4}=1, x_{2}+x_{3}+x_{5}=1, x_{3}+x_{1}+x_{6}=1, x_{4}+x_{5}+x_{6}=1
$$

The only face of a polytope of quasiadjunction which belongs to a hyperplane $H_{k}: x_{1}+x_{2}+x_{3}+x_{4}+x_{5}+x_{6}=k, k \in \mathbf{Z}$ is formed by set of solutions of the system of all 4 equations (3.3.1). This face belongs to $\mathrm{H}_{2}$ and is the only contributing face. Hence the irregularity is equal to $N \cdot \operatorname{dim} H^{1}(\mathcal{J}(6-3-2))$ where $N$ is the number of solutions (3.3.1) of the form $x_{i}=\frac{j}{n}$. To calculate $\operatorname{dim} H^{1}(\mathcal{J}(6-3-2))$ notice that 4 triple points form a complete intersection of two quadrics. This yields $H^{1}(\mathcal{J}(1))=H^{2}(\mathcal{O}(-3))=\mathbf{C}$.

It follows from (3.3.1) that the only essential torus is a component of subgroup:

$$
t_{1} t_{2} t_{4}=1, t_{2} t_{3} t_{5}=1, t_{1} t_{3} t_{6}=1, t_{4} t_{5} t_{6}=1
$$

This subgroup has two connected components:

$$
\left(u, v, u^{-1} v^{-1}, u^{-1} v^{-1}, u, v\right),\left(-u,-v,-u^{-1} v^{-1}, u^{-1} v^{-1}, u, v\right), \quad u, v \in \mathbf{C}^{*}
$$

The second component is a translation of the first by $(1,1,1,-1,-1,-1)$, a point of order 2. Since (3.3.1) admits an integral solution image under the exponential map of the contributing face does contains trivial character and hence the subgroup in (3.3.3) is the essential torus.

There are also 4 nonessential tori corresponding to each of triple points:

$$
\begin{aligned}
& t_{1} t_{2} t_{3}=1, t_{i}=1, i \neq 1,2,3 \\
& t_{5} t_{2} t_{3}=1, t_{i}=1, i \neq 5,2,3 \\
& t_{4} t_{6} t_{3}=1, t_{i}=1, i \neq 4,6,3 \\
& t_{4} t_{5} t_{6}=1, t_{i}=1, i \neq 4,5,6
\end{aligned}
$$

Let us consider the abelian cover of $\mathbf{C}^{2}$ corresponding to the homomorphism $H_{1}\left(\mathbf{C}^{2}-C\right) \rightarrow(\mathbf{Z} / n \mathbf{Z})^{6} / \mathbf{Z} / n \mathbf{Z}$ (embedding of the quotiented subgroup is diagonal). Then each of five tori contributes the same number into irregularity 
equal to $\frac{n^{2}-3 n+2}{2}$, i.e. the irregularity of the abelian cover of $\mathbf{C P}^{2}$ is $5 \frac{n^{2}-3 n+2}{2}$ (e.g., for $n=5$ the irregularity is 30, cf. [I]).

Example 3. Let us calculate the characteristic varieties of the union of 9 lines which are dual to nine inflection points on a non singular cubic curve $C \subset \mathbf{P}^{2}(\mathbf{C})$. This arrangement in $\mathbf{P}^{2^{*}}(\mathbf{C})$ has 12 triple points corresponding to 12 lines determined by the pairs of the inflection points of $C$. One can view inflection points of $C$ as the points of $\mathbf{F}_{3}^{2}\left(\mathbf{F}_{3}\right.$ is the field with 3 elements) i.e. as the points of the affine part in a projective plane $\mathbf{P}^{2}\left(\mathbf{F}_{3}\right)$. The triple points of this arrangement then can be viewed as lines in $\mathbf{P}^{2}\left(\mathbf{F}_{3}\right)$ different from the line at infinity (i.e. the complement to the chosen affine plane). In dual picture one identifies triple points of this arrangement with points of the dual plane $\mathbf{P}^{2^{*}}\left(\mathbf{F}_{3}\right)$ different from a fixed point $P$ corresponding to the line at infinity. Then the lines of this arrangement in $\mathbf{P}^{2^{*}}(\mathbf{C})$ are identifies with the lines in $\mathbf{P}^{2 *}\left(\mathbf{F}_{3}\right)$ not passing through the fixed point $P$.

Each essential component corresponds to a collection of vertices $\mathcal{S}$ (cf. (2.6.4), example 2). The structure of the system of equations (2.6.1) shows that $\frac{|\mathcal{S}|}{k}=\frac{r}{m}=3$. Hence one has either:

a) $|\mathcal{S}|=3, k=1$ or

b) $|\mathcal{S}|=6, k=2$ or

c) $|\mathcal{S}|=9, k=3$ or

d) $|\mathcal{S}|=12, k=4$.

Cases a) and b) will not define non empty tori since in this case $r^{2}>9|\mathcal{S}|$ (cf. corollary 4.1).

In the case c) each collection $\mathcal{S}$ is determined by one of 4 choices of a line $\ell$ through $P$ and consists of 9 points in $\mathbf{P}^{2 *}\left(\mathbf{F}_{3}\right)$ in the complement to the chosen line. In this case the corresponding homogeneous system has rank 7 i.e. a 2-dimensional space of solutions. Moreover, $\operatorname{dim} H^{1}\left(\mathbf{P}^{2 *}(\mathbf{C}), \mathcal{I}(9-3-\right.$ $3))=1$ since the points on $\mathbf{P}^{2^{*}}(\mathbf{C})$ corresponding to 9 points in $\mathbf{P}^{2^{*}}\left(\mathbf{F}_{3}\right)$ in the complement to a line $p \subset \mathbf{P}^{2 *}\left(\mathbf{F}_{3}\right)$ form a complete intersection of two cubics. These cubics formed by the unions of triples of lines in $\mathbf{P}^{2^{*}}(\mathbf{C})$ corresponding to triple of lines in $\mathbf{P}^{2 *}\left(\mathbf{F}_{3}\right)$ passing through a point of $\ell$. Indeed, for a given $P_{1}, P_{2} \in \ell$ and a point $Q$ on $\mathbf{P}^{2^{*}}\left(\mathbf{F}_{3}\right)$ outside of $\ell$, there are exactly 2 lines in $\mathbf{P}^{2 *}\left(\mathbf{F}_{3}\right)$ intersecting at this point and passing respectively through $P_{1}$ and $P_{2}$. The same incidence relation is valid on $\mathbf{P}^{2^{*}}(\mathbf{C})$.

In the case d) the homogeneous system has rank 9 , i.e. the corresponding system does not define a torus. 
Non essential tori correspond to subarrangements with number of lines divisible by $m=3$ (cf. 2.6.3). There are 12 triples of lines corresponding to each of triple points each defining a 2-torus. A collection of 6 lines should have 4 triple points but the arrangement of this example does not contain such subarrangements.

Therefore we have 16 2-dimensional tori. In the abelian cover of $\mathbf{C}^{2}$ which sends each generator of $H_{1}\left(\mathbf{C}^{2}-\mathcal{C}\right)$ to a generator of $\mathbf{Z} / n \mathbf{Z}$ contributing tori are the essential torus of this arrangement and subtori corresponding to subarrangements formed by triple of lines defined by the triple points. Each torus contributes $\frac{(n-1)(n-2)}{2}$ to the Betti number i.e. the total Betti number of this cover is $16 \times \frac{(n-1)(n-2)}{2}$. These tori can be explicitly described as follows. Defining equations of non essential tori are products of 3 generators $t_{i}$ 's corresponding to a triple of points in $\mathbf{F}_{3}^{2}$ belonging to a line with the rest of $t_{i}$ is 1 . Each of essential tori is given by 9 equations $t_{i} t_{j} t_{k}=1$ where $(i, j, k)$ are the triples of points $\mathbf{F}_{3}^{2}$ (which interpreted as the lines of the arrangement) which belong to lines not passing through a fixed point at infinity.

If the point at infinity is $(1,-1,0)$, then the lines not passing through it are: $x+z=0, x-z=0, x=0, y=0, x-y=0, x-y+z=0, x-y-z=$ $0, y+z=0, y-z=0$ i.e. the corresponding torus satisfies:

$$
\begin{gathered}
t_{20} t_{21} t_{22}=1, t_{10} t_{11} t_{12}=1, t_{00} t_{01} t_{02}=1, t_{00} t_{10} t_{20}=1, t_{00} t_{11} t_{22}=1 \\
t_{01} t_{12} t_{20}=1, t_{02} t_{10} t_{21}=1, t_{02} t_{12} t_{22}=1, t_{01} t_{11} t_{21}=1
\end{gathered}
$$

where the points of the complement to $z=0$ (i.e. the lines in $\mathbf{P}^{2 *}(\mathbf{C})$ ) are labeled as:

$$
(0,0),(0,1),(0,2),(1,0),(1,1),(1,2),(2,0),(2,1),(2,2)
$$

The corresponding torus can be parameterized as

$$
\begin{gathered}
t_{00}=t, t_{01}=s, t_{02}=t^{-1} s^{-1}, t_{10}=s, t_{11}=t^{-1} s^{-1}, t_{12}=t, \\
t_{20}=t^{-1} s^{-1}, t_{21}=t, t_{22}=s .
\end{gathered}
$$

The equations for other essential tori, corresponding to choices of the point at infinity as respectively: $(1,1,0),(1,0,0),(0,1,0)$ can be obtained from (3.3.4) by applying linear transformation to the indices which takes 
$(1,-1,0)$ to respective point. For $(x, y) \rightarrow(x,-y)$ which takes $(1,-1,0)$ to $(1,1,0)$ we obtain:

$$
\begin{gathered}
t_{20} t_{22} t_{21}=t_{10} t_{12} t_{11}=t_{00} t_{02} t_{01}=t_{00} t_{10} t_{20}=t_{00} t_{12} t_{21}= \\
t_{02} t_{11} t_{20}=t_{01} t_{10} t_{22}=t_{01} t_{11} t_{21}=t_{02} t_{12} t_{21}=1
\end{gathered}
$$

For $(x, y) \rightarrow(x, x+y)$ which takes $(1,-1,0)$ to $(1,0,0)$ we obtain:

$$
\begin{gathered}
t_{22} t_{20} t_{21}=t_{11} t_{12} t_{10}=t_{00} t_{01} t_{02}=t_{00} t_{11} t_{22}=t_{00} t_{12} t_{21}= \\
t_{01} t_{10} t_{20}=t_{02} t_{11} t_{20}=t_{02} t_{10} t_{21}=t_{01} t_{12} t_{20}=1
\end{gathered}
$$

For $(x, y) \rightarrow(x+y, y)$ which takes $(1,-1,0)$ to $(0,1,0)$ we have:

$$
\begin{gathered}
t_{20} t_{01} t_{12}=t_{10} t_{21} t_{02}=t_{00} t_{11} t_{22}=t_{00} t_{10} t_{20}=t_{00} t_{21} t_{12} \\
t_{11} t_{01} t_{20}=t_{22} t_{10} t_{01}=t_{22} t_{02} t_{12}=t_{11} t_{21} t_{01} .
\end{gathered}
$$

Example 4. Let us consider the curve of degree 4 which has one ordinary point of multiplicity 4. Faces of the polytopes of quasiadjunction are $H_{1}$ : $x_{1}+x_{2}+x_{3}+x_{4}=1$ (resp. $\left.H_{2}: x_{1}+x_{2}+x_{3}+x_{4}=2\right)$. The number of points $\left(\frac{i_{1}}{n}, \frac{i_{2}}{n}, \frac{i_{3}}{n}, \frac{i_{4}}{n}\right)$ on $H_{1}$ (resp. $\left.H_{2}\right)$ is $\frac{(n-1)(n-2)(n-3)}{6}$ (resp. $\frac{1}{3}(n-1)\left(2 n^{2}-\right.$ $4 n+3))$. The ideal corresponding to the polytope of quasiadjunction with the face $H_{1}$ (resp. $H_{2}$ ) is $\mathcal{M}^{2}$ (resp. $\mathcal{M}$ the maximal ideal of the local ring) and the level of the supporting face $H_{1}$ (resp. $H_{2}$ ) is 1 (resp. 2). Moreover $\operatorname{dim} H^{1}\left(\mathbf{P}^{2}, \mathcal{J}_{\mathcal{M}^{3-l}}(4-3-l)\right.$ ) is 2 (resp. 1 ) for $l=1$ (resp. for $l=2$ ). Hence the irregularity of the cover corresponding to homomorphism $H_{1}\left(\mathbf{P}^{2}-\cup_{i=1,2,3,4} L_{i}\right) \rightarrow \mathbf{Z} / n \mathbf{Z}$ is equal to

$$
\begin{gathered}
2 \times \frac{1}{6}(n-1)(n-2)(n-3)+\frac{1}{3}(n-1)\left(2 n^{2}-4 n+3\right)+4 \frac{1}{2}(n-1)(n-2)= \\
(n-1)\left(n^{2}-n-1\right) .
\end{gathered}
$$

This implies that the characteristic variety in this case is just

$$
t_{1} t_{2} t_{3} t_{4}=1
$$

The latter contains $(n-1)^{3}-(n-1)(n-2)=(n-1)\left(n^{2}-3 n+3\right)$ points with coordinates in $\mu_{n}$ and the Betti number of the branched cover from Sakuma's 
formula is equal to $2(n-1)\left(n^{2}-3 n+3\right)+4(n-1)(n-2)=2(n-1)\left(n^{2}-n-1\right)$.

Example 5. Let us consider the arrangement formed by 12 lines which compose 4 degenerate fibers in a Hesse pencil of cubics formed by a non singular cubic curve and its Hessian. For example one can take the following pencil:

$$
x^{3}+y^{3}+z^{3}-3 \lambda x y z=0
$$

This arrangement has 9 points of multiplicity 4 (inflection points of non singular cubic). In $\mathbf{C}^{* 12}$ there are 10 tori of dimension 3 which are defined by 9 quadruples of lines corresponding to 9 quadruple points and one 3 torus corresponding to the whole configuration. Contribution into the first Betti number an abelian cover also comes from 94 tori of dimension 2: 2-tori corresponding to triples of lines forming each of 9 quadruple points (total 36 2-tori), 2-tori corresponding to configurations of 9 lines formed by triples of 4 special fibers of the pencil (total 4 2-tori) and 54 2-tori corresponding to configurations of 6 lines passing through 4 inflection points no three of which belong to a line (since the choice of 4 points must be made among points of affine space over $\mathbf{F}^{3}$ the ordered collection can be made in $9 \times 8 \times 6 \times 3$ way and $54=9 \cdot 8 \cdot 6 \cdot 3 / 24)$. In particular, the irregularity of the cover with the Galois group $(\mathbf{Z} / 3 \mathbf{Z})^{2}$ is equal to 154 (cf. [I]). Indeed the contribution of each 2-torus into the first Betti number is 2 and in the case of 3-tori the

contribution is 6 , since the 3 -torus contains 6 points with coordinates $\frac{i}{3}$. Since the depth of 3 -tori is 2 the first Betti number is equal to $6 \times 10 \times 2+94 \times 2$.

\section{The structure of characteristic varieties of algebraic curves.}

In this section we describe sufficient conditions for the vanishing of cohomology of linear systems which appear in description of characteristic varieties given in section 3. This, therefore, yields conditions for absence of essential components. In the cyclic case one obtains triviality of Alexander polynomial. 


\subsection{Absence of characteristic varieties for curves with small number of singularities.}

\subsection{1}

Theorem 4.1.1 Let $\mathcal{C}$ be a plane curve as above. Suppose that $\rho: Y \rightarrow \mathbf{P}^{2}$ is obtained by a sequence of blow ups such that the proper preimage $\tilde{\mathcal{C}}$ of $\mathcal{C}$ in $Y$ has only normal intersection with the exceptional set and satisfies $\tilde{\mathcal{C}}^{2}>0$. Then $\mathcal{C}$ has no essential characteristic subvarieties.

Corollary 4.1.2 1. Let $\mathcal{C}$ be an irreducible curve which has ordinary cusps and nodes as the only singularities. If the number of cusps is less than $\frac{d^{2}}{6}$ then the Alexander polynomial of $\mathcal{C}$ is equal to 1.

2. Let $\mathcal{H}$ be an arrangement consisting of d lines and which has $N$ points of multiplicity $m$. Let $l(\delta)$ be the level of a face of quasiadjunction for the complement to $\mathcal{H}$. If $d^{2}>m^{2} N$ then the superabundance is zero for the system of curves of degree $d-3-l(\delta)$ which local equations belong to the ideal of quasiadjunction corresponding to $\delta$ at the points of multiplicity $m$.

Remark. One can compare corollary 1 with Nori's results (cf. [N]). The latter yields that the fundamental group of the complement to a curve of degree $d$ with $\delta$ nodes and $\kappa$ cusps is abelian if $d^{2}>6 \kappa+2 \delta$ while a weaker inequality $d^{2}>6 \kappa$ yields the triviality of the Alexander polynomial. For example, for the branching curve of a generic projection of a smooth surface of degree $N$ in $\mathbf{P}^{3}$ one has $d^{2}>6 \kappa$ for $N>4$ but $d^{2}<6 \kappa+2 \delta$ for $N>2$. The fundamental groups of these curves are non abelian for $N>2$ and the Alexander polynomial for $N=3,4$ is equal to $t^{2}-t+1$ (cf. [L2]).

Proof of the theorem. We should show that for any contributing face of quasiadjunction $\delta$ we have $\operatorname{dim} H^{1}\left(\mathbf{P}^{2}, \mathcal{A}_{\delta}\left(\Sigma d_{i}-3-l(\delta)\right)\right)=0$. If $\rho: Y \rightarrow \mathbf{P}^{2}$ is a blow up of $\mathbf{P}^{2}$, satisfying conditions of the theorem, then we have:

$$
\mathcal{A}_{\delta}\left(\Sigma_{i} d_{i}-3-l(\delta)\right)=\rho_{*}\left(\omega_{Y} \otimes \mathcal{O}_{Y}(\gamma \tilde{\mathcal{C}}) \otimes \mathcal{O}_{Y}\left(\Sigma \epsilon_{k} E_{k}\right)\right)
$$

for some rational $\gamma>0$ and $0 \leq \epsilon<1$. More precisely, $\gamma=\frac{1}{\Sigma_{i} d_{i}} \cdot \Sigma_{i} d_{i}(1-$ $\left.\frac{j_{i}+1}{m_{i}}\right)$ for some choice of $\left(\ldots, \frac{j_{i}+1}{m_{i}}, \ldots\right)$ belonging to the face $\delta$ (with $\epsilon_{k}$ a priori 
depending on this choice). Indeed, from (2.3.6) and the discussion after, the multiplicity $f_{k}(\phi)$ along an exceptional curve $E_{k}$ of the pull back on $Y$ of a germ in the ideal of quasiadjunction with parameters $\left(j_{1}, . ., j_{r} \mid m_{1}, \ldots, m_{r}\right)$ such that $\left(\frac{j_{1}+1}{m_{1}}, \ldots, \frac{j_{r}+1}{m_{r}}\right) \in \delta$ satisfies:

$$
f_{k}(\phi) \geq\left[\Sigma_{i} a_{k, i}\left(1-\frac{j_{i}+1}{m_{i}}\right)-c_{k}\right]
$$

Hence

$$
A_{\delta}=\rho_{*}\left(\otimes_{k} \mathcal{O}_{Y}\left(\left(c_{k}-\left[\Sigma_{i} a_{k, i}-a_{k, i}\left(\frac{j_{i}+1}{m_{i}}\right)\right]\right) E_{k}\right)\right)
$$

We have: $l(\delta)=\Sigma_{i} d_{i} \frac{j_{i}+1}{m_{i}}$ and $\mathcal{O}_{\mathbf{P}^{2}}\left(C_{i}\right)=\mathcal{O}_{\mathbf{P}^{2}}\left(d_{i}\right)$. Therefore

$$
\begin{gathered}
A_{\delta}\left(\Sigma_{i} d_{i}-3-l(\delta)\right)= \\
\rho_{*}\left(\otimes_{k} \mathcal{O}_{Y}\left(\left(c_{k}-\left[\Sigma_{i} a_{k, i}-a_{k, i}\left(\frac{j_{i}+1}{m_{i}}\right)\right]\right) E_{k}\right)\right) \otimes \mathcal{O}_{\mathbf{P}^{2}}(-3) \otimes \mathcal{O}_{\mathbf{P}^{2}}\left(\Sigma_{i} C_{i}\left(1-\frac{j_{i}+1}{m_{i}}\right)\right)
\end{gathered}
$$

Since

$$
\omega_{Y}=\otimes_{k} \mathcal{O}_{Y}\left(c_{k} E_{k}\right) \otimes \rho^{*}\left(\mathcal{O}_{\mathbf{P}^{2}}(-3)\right)
$$

and

$$
\otimes_{k} \mathcal{O}_{Y}\left(a_{k, i}\left(1-\frac{j_{i}+1}{m_{i}}\right) E_{k}\right) \otimes \mathcal{O}(\tilde{\mathcal{C}})^{\frac{d_{i}}{\Sigma d_{i}}}=\rho^{*}\left(\mathcal{O}_{\mathbf{P}^{2}}\left(C_{i}\left(1-\frac{j_{i}+1}{m_{i}}\right)\right)\right)
$$

(because $\mathcal{O}_{\mathbf{P}^{2}}\left(C_{i}\right)=\mathcal{O}_{\mathbf{P}^{2}}(1)^{d_{i}}=\mathcal{O}_{\mathbf{P}^{2}}(\mathcal{C})^{\frac{d_{i}}{\Sigma_{i} d_{i}}}$ ), we see that (4.1.1.3) yields (4.1.1) with $\epsilon_{k}=\left\{\sum_{i} a_{k, i}\left(1-\frac{j_{i}+1}{m_{i}}\right)\right\}$ where $\{x\}=x-[x]$ is the fractional part.

The Kawamata-Viehweg vanishing theorem (cf. for example [Ko]) implies that the cohomology of the sheaf $\omega_{Y} \otimes \mathcal{O}_{Y}(\gamma \tilde{\mathcal{C}}) \otimes \mathcal{O}_{Y}\left(\sum \epsilon_{k} E_{k}\right)$ is trivial in positive dimensions if $\tilde{\mathcal{C}}$ is big and nef. But this follows from the assumptions of the theorem. Finally, the exact sequence $0 \rightarrow E_{2}^{1,0} \rightarrow H^{1}(Y, \mathcal{F})$ of lower degree terms in the Leray spectral sequence $H^{p}\left(\mathbf{P}^{2}, R^{q} \rho_{*} \mathcal{F}\right) \Rightarrow H^{p+q}(Y, \mathcal{F})$ for the sheaf $\mathcal{F}=\omega_{Y} \otimes \mathcal{O}_{Y}(\gamma \tilde{\mathcal{C}}) \otimes \mathcal{O}_{Y}\left(\sum \epsilon_{k} E_{k}\right)$ yields $H^{1}\left(\mathbf{P}^{2}, \mathcal{A}_{\delta}\left(\Sigma_{i} d_{i}-3-\right.\right.$ $l(\delta)))=0$.

Proof of the Corollary. For each blow up at an ordinary point of multiplicity $m$ of a curve $C$ we have $\tilde{C}^{2}=C^{2}-m^{2}$ where $\tilde{C}$ is the proper preimage of $C$. Hence $\mathcal{O}(\tilde{\mathcal{H}})$ is big if $d^{2}>m^{2} N$. The case of ordinary cusps is similar. 


\subsubsection{Dimensions of components of characteristic varieties.}

This theorem imposes restrictions on the dimensions of the contributing faces and hence on the dimensions of characteristic varieties. For example, let us consider an arrangement of lines with at most triple points as singularities. Then each contributing face is the intersection of hyperplanes defining the only local polytope of an ordinary triple point. These hyperplanes are given by the equations of the form $x_{i}+x_{j}+x_{k}=1$ where $(i, j, k)$ are the indices corresponding to the lines through the triple point. The matrix of this system therefore has the property that in each row only 3 non zero entries are equal to 1 , any two rows have at most one non zero entry in the same column and the number of rows is at least $\frac{d^{2}}{9}$ (since by the corollary only in this case one can get a contributing face with $H^{1} \neq 0$ ). In particular, the number of non

zero entries in the matrix is at least $\frac{d^{2}}{3}$. The rank of this system is at least $\frac{d}{3}$. Indeed, the matrix contains a column with at least $\frac{d}{3} 1$ 's. On the other hand, if a column $s$ contains $k$ non zero entries in rows $v_{1}, . ., v_{k}$, then these rows are linearly independent since the left hand side of a relation $\Sigma \lambda_{i} v_{i}=0$ has $\lambda_{i}$ as the entry of the column different from the $s$-th and in which $v_{i}$ has a non zero entry. In particular in the arrangement of $d$ lines, the dimension of characteristic variety is at most $\frac{2 d}{3}$. For the components containing a trivial character, the dimension is at most 2 , since by $[\mathrm{Ar}]$ such component induces the map onto $\mathbf{P}^{1}$ minus three point such that pull backs of rank one local systems from the latter form the component (cf. footnote in section 1 and [LY]).

Problem. Let $m$ be the maximal number of components which meet at a singular point of $\mathcal{C}$. Is it true that the dimension of each component of the characteristic variety of $\mathcal{C}$ is at most $m-1$ ?

\subsection{Translations of the tori forming characteristic va- rieties and the degrees of irreducible components of $\mathcal{C}$}

Theorem 4.2.1 Let $a_{\delta}$ be g.c.d. of non zero minors of maximal order in a system of equations with integer coefficients which set of solutions contains 
a face of quasiadjunction $\delta$ with the dimension of the set of solutions equal to $\operatorname{dim} \delta$. Let $d_{i}=\operatorname{deg} C_{i}$.

a) Each irreducible essential component of CharC, belongs to a coset of a subtorus of $\operatorname{Hom}\left(H_{1}\left(\mathbf{C}^{2}-\mathcal{C}\right), \mathbf{C}^{*}\right)$ of order dividing $a_{\delta}$. In particular each irreducible essential component belongs to a coset of order equal to the order of the face (cf. 2.4.2 and 2.6.2).

b) Each essential component belongs to a codimension one subtorus of $\operatorname{Hom}\left(\mathrm{H}_{1}\left(\mathbf{C}^{2}-\right.\right.$ $\left.\mathcal{C}), \mathbf{C}^{*}\right)$ translated by a point of the order dividing $\varrho=$ g.c.d. $\left(d_{1}, \ldots, d_{r}\right)$.

Proof. Any component of a characteristic variety is a Zariski closure of the image under the exponential map of a contributing face $\delta$ with the equation $d_{1} x_{1}+\ldots+d_{r} x_{r}=l(\delta)$. This yields b) (it also follows from Cor.3.3 from [L3]).

It follows from theorem $3.1 \mathrm{c}$ ) that irreducible essential component is a connected component of the subgroup of $\operatorname{Hom}\left(H_{1}\left(\mathbf{C}^{2}-\mathcal{C}\right), \mathbf{C}^{*}\right)$ belongs to a subgroup

$$
\chi_{1}=\ldots=\chi_{r}=1
$$

where $\chi_{i}$ is the character of $\operatorname{Hom}\left(H_{1}\left(\mathbf{C}^{2}-\mathcal{C}\right), \mathbf{C}^{*}\right)$ having form $\exp \left(L_{i}\right)$ where $L_{i}$ is the form with integer coefficients such that $L_{i}=l_{i}, l_{i} \in \mathbf{Z}$ are the equations defining the face of quasiadjunction. The order of the group of cosets of the group (4.2.1.1) by its connected component of identity is the order of the torsion of its group of characters. The latter is $\operatorname{Char}\left(\operatorname{Hom}\left(H_{1}\left(\mathbf{C}^{2}-\mathcal{C}\right), \mathbf{C}^{*}\right)\right) /\left(\chi_{1}, \ldots \chi_{r}\right)$. This yields a $)$.

\subsubsection{Linear systems corresponding to different faces of quasiad- junction.}

Another byproduct of results in section 3 is equality of superabundances of linear systems of curves defined by rather different local conditions.

Proposition 4.2.2 a) Let $\delta$ and $\delta^{\prime}$ be two faces of global polytopes quasiadjunction such that the Zariski closures of $\exp (\delta)$ and $\exp \left(\delta^{\prime}\right)$ coincide. Then if $\delta$ is a contributing face then $\delta^{\prime}$ is also contributing and $H^{1}\left(\mathcal{A}_{\delta}(d-3-l(\delta))=\right.$ $H^{1}\left(\mathcal{A}_{\delta^{\prime}}\left(d-3-l\left(\delta^{\prime}\right)\right)\right.$.

b) Let $\alpha \in \mathbf{Q}$ is such that $\alpha \cdot$ g.c.d $\left(d_{1}, \ldots, d_{r}\right)$ is the level of a face of quasiadjunction $\delta$ and $\sigma \in \operatorname{Gal}(\mathbf{Q}(\exp (2 \pi i \alpha)) / \mathbf{Q})$ such that $\sigma(\exp (2 \pi i \alpha))=\exp (2 \pi i \beta)$ 
with $0<\beta<1$. Then $\beta$ is equal to $\frac{l\left(\delta^{\prime}\right)}{\text { g.c.d }\left(d_{1}, . ., d_{r}\right)}$ for some face of quasiadjunction $\delta^{\prime}$ and $H^{1}\left(\mathcal{A}_{\delta}\left(\Sigma_{i} d_{i}-3-l(\delta)\right)\right)=H^{1}\left(\mathcal{A}_{\delta^{\prime}}\left(d-3-l\left(\delta^{\prime}\right)\right)\right)$.

Proof. a) Since Zariski closures of $\exp (\delta)$ and $\exp \left(\delta^{\prime}\right)$ are the same the corresponding to $\delta$ and $\delta^{\prime}$ components of the characteristic variety are the same. If the depth of this component of characteristic variety is $i$, then the dimension of each of the cohomology group in the statement equals to $i$ and the result follows.

b) Since i-th characteristic variety is defined over $\mathbf{Z}$ the Galois $\operatorname{group} \operatorname{Gal}(\overline{\mathbf{Q}} / \mathbf{Q})$ acts on the set of its irreducible components. Since irreducible component corresponding to $\delta$ is a translation by $\exp (2 \pi \sqrt{-1} \alpha)$ of a subgroup of $H^{1}\left(\mathbf{C}^{2}-\mathcal{C}, \mathbf{C}^{*}\right)$ defined over $\mathbf{Z}$, it follows that $\sigma$ takes the component corresponding to $\delta$ into translation of the same subgroup by $\exp (2 \pi \sqrt{-1} \beta)$. This translation is a Zariski closure of $\exp \left(2 \pi \sqrt{-1} \delta^{\prime}\right)$ for some face $\delta^{\prime}$. It does satisfy the conclusions of $b$ ).

\subsubsection{Remarks.}

1. For irreducible curves the order of each face of quasiadjunction is a root of a local Alexander polynomial. So the divisibility theorem from [L1] is a special case of 4.2.1.

2. One of the consequences of 4.2.1 is a non trivial restriction on an abstract group which is necessary to satisfy in order that the group can be realized as the fundamental group of an arrangement. For example $t_{1} \cdots t_{r}=-1$ cannot be a component of characteristic variety of arrangement of $r$ lines since it is cannot belong to an intersection of subgroups of $\mathbf{C}^{* r}$.

3. As an illustration to $4.2 .2 \mathrm{~b}$ ), let us consider an irreducible curve of degree $d$ with singularities locally isomorphic to singularity $x^{2}=y^{5}$. If the linear system consisting of curves of degree $d-3-\frac{d}{10}$ with local equations belonging to ideals of quasiadjunction of all singular points corresponding to the constant of quasiadjunction $\frac{1}{10}$ is superabundant, then the linear system of curves of degree $d-3-\frac{3 d}{10}$ with local equations in the ideals of quasiadjunction corresponding to $\frac{3}{10}$ is also superabundant and the superabundances are equal.

4. An example of faces of quasiadjunction with the same Zariski closure of the images of the exponential map as in a) of the proposition is given 
by $x_{1}+\ldots x_{m}=i, x_{1}+. .+x_{m}=j, 0<i, j \leq m-2$ which are the faces of quasiadjunction for the complement to $m$ lines through a point (cf. 2.5 example 3).

\section{Resonance conditions for rank one local sys- tems on complements to line arrangements.}

\subsection{Complexes associated with arrangements.}

Let $\mathcal{L}=\cup_{i=1, \ldots, r} L_{i}$ be an arrangement of lines in $\mathbf{C}^{2}$. We shall assume for convenience (cf. (1.2.3)) that the line at infinity is transversal to all lines in $\mathcal{L}$. Let $l_{i}(x, y)=0$ be the equation of $L_{i}$ and $\eta_{i}=\frac{1}{2 \pi i} \frac{d l_{i}}{l_{i}}$. Let $A^{i}(i=0,1,2)$ be the subspace generated by the forms $\eta_{j_{1}} \wedge \ldots \wedge \eta_{j_{i}}$ in the space $H^{0}\left(\Omega^{i}(* \mathcal{L})\right)$ of meromorphic forms with poles along $\mathcal{L}$. Let

$$
\omega=\Sigma \eta_{i} \cdot s_{i}, s_{i} \in \mathbf{C}
$$

The exterior product with $\omega$ defines the complex:

$$
A_{\omega}^{\bullet}: 0 \rightarrow A^{0} \rightarrow A^{1} \rightarrow A^{2} \rightarrow 0
$$

If $s_{i}=0,(i=1, \ldots, r)$, then the cohomology groups of $A^{\bullet}$ are isomorphic to the cohomology groups of $\mathbf{C}^{2}-\mathcal{L}(\mathrm{cf}$. $[\mathrm{Br}])$. On the other hand, the collection $s_{*}=\left(s_{1}, \ldots, s_{r}\right)$ defines the map $\pi_{1}\left(\mathbf{C}^{2}-\mathcal{L}\right) \rightarrow \mathbf{C}^{*}$ which sends $\gamma_{i}$ (cf. (1.1)) to $\exp \left(2 \pi \sqrt{-1} s_{i}\right)$ and hence the local system which we shall denote $\mathcal{A}_{s_{*}}$. A theorem from [ESV] (p.558), in the case of line arrangements, asserts that

$$
H^{i}\left(\mathcal{A}_{s_{*}}\right)=H^{i}\left(A_{\omega}^{\bullet}\right)
$$

provided the following non resonance condition is satisfied. For any point singular point $P$ of $\mathcal{L}$ of multiplicity $m>2$, if the lines through $P$ are $l_{i_{1}}, \ldots, l_{i_{m}}$, then

$$
s_{i_{1}}+\ldots+s_{i_{m}} \neq n \in \mathbf{N}-0
$$

Theorem 5.1.1 The isomorphism (5.1.3) takes place, provided

$$
\left(\exp \left(2 \pi i s_{1}\right), \ldots, \exp \left(2 \pi i s_{r}\right)\right)
$$

does not belong to the characteristic variety Char $_{1}$ of $\mathbf{C}^{2}-\mathcal{L}$. 


\subsection{Remarks.}

1. It is easy to construct examples of local systems for which (5.1.4) is violated but for which (1.5.3) takes place. Indeed, the image under the exponential map onto the torus $\mathbf{C}^{* r}$ of those $\left(s_{1}, \ldots, s_{r}\right)$ which violate (5.1.4) is a union of codimension 1 tori in $\mathbf{C}^{* r}$. On the other hand, the characteristic varieties typically have rather small dimension relative to $r$ (cf. (3.3) and (4.1)).

2. For arrangements of arbitrary dimension in $\mathbf{C}^{n}$ (with $l_{i}$ denoting the equations of hyperplanes of the arrangement, rather than lines) we have

$$
H^{1}\left(A^{\bullet}(\mathcal{L}), \omega\right)=0
$$

provided condition (5.1.5) of the theorem is met. Indeed, for a generic plane $H \subset \mathbf{C}^{n}$ the map $A^{i}(\mathcal{L}) \rightarrow A^{i}(\mathcal{L} \cap H)$ induced by inclusion is isomorphism for $i=0,1$ and injective for $i=2$. The latter follows from the Lefschetz theorem since $A^{i}(\mathcal{L})=H^{i}\left(\mathbf{C}^{n}-\mathcal{L}\right)$ by $[\mathrm{Br}]$. This yields the isomorphism of cohomology of the complexes $A^{\bullet}$ for $\mathcal{L}$ and $\mathcal{L} \cap H$ and hence (5.2.1). Therefore the theorem from (5.1) (and also from (5.4)) holds for arbitrary arrangements.

\subsection{Proof}

We will derive this theorem from the following:

a) $H^{0}\left(A_{\omega}^{\bullet}\right)=H^{1}\left(A_{\omega}^{\bullet}\right)=0$

b) The euler characteristics of both $A_{\omega}^{\bullet}$ and $\mathcal{A}_{s_{*}}$ are equal to $e\left(\mathbf{C}^{2}-\mathcal{L}\right)$.

To show b) note that the euler characteristic of $A^{\bullet}$ is $e\left(\mathbf{C}^{2}-\mathcal{L}\right)$ by $[\mathrm{Br}]$ and for $H^{i}\left(\mathcal{A}_{s_{*}}\right)$ this can be seen by looking at the cochain complex of $\mathcal{A}_{s_{*}}$, i.e. (here $\chi_{s_{*}}$ is the character of the fundamental group defining the local system A)

$$
C^{i}\left(\left(\mathbf{C}^{2}-\mathcal{L}\right) \otimes_{\chi_{s_{*}}} \mathbf{C}\right)=\mathbf{C}^{b_{i}} \otimes \mathbf{C}\left[H_{1}\left(\mathbf{C}^{2}-\mathcal{L}\right)\right] \otimes_{\chi_{s_{*}}} \mathbf{C}
$$

since the multiplicity of a representation in the regular representation of an abelian group is 1 .

On the other hand a) follows since $\operatorname{dim} H^{1}\left(A^{\bullet}, \omega\right) \leq \operatorname{dim} H^{1}\left(\mathcal{A}_{s_{*}}\right)$ (cf. $[\mathrm{LY}])$ and the latter group is trivial if (5.1.5) is satisfied.

Now the theorem 5.1 is a consequence of a),b) and (1.4.1.1). 


\subsection{Complexes (5.1.2) with non vanishing cohomology.}

Complexes $A^{\bullet}$ for a fixed arrangement $\mathcal{L}$ are parameterized by the space $H^{0}\left(\mathbf{P}^{2}, \Omega^{1}\left(\log \left(\mathcal{L} \cup L_{\infty}\right)\right)=H^{1}\left(\mathbf{C}^{2}-\mathcal{L}, \mathbf{C}\right)\right.$. Let

$$
\mathcal{V}_{i}=\left\{\omega \in H^{0}\left(\mathbf{P}^{2}, \Omega^{1}\left(\log \left(\mathcal{L} \cup L_{\infty}\right)\right)\right) \mid H^{1}\left(A^{\bullet}, \omega\right) \geq i\right\}
$$

Theorem 5.4.1 $\mathcal{V}_{i}$ is a union of linear space of dimension $i+1$. There is the one to one correspondence between these linear spaces and irreducible components of the characteristic variety $V_{i}$ containing the trivial character which have a positive dimension. For such an irreducible component of $\mathcal{V}_{i}$ the map which assigns to a form $\omega$ the point $\left(\ldots, \exp \left(2 \pi i \operatorname{Res}_{L_{i}} \omega\right), \ldots\right)$ in $H^{1}\left(\pi_{1}\left(\mathbf{C}^{2}-\mathcal{L}\right), \mathbf{C}^{*}\right)$ (using identification (1.4.1)) is the universal cover of the corresponding component of $V_{i}$.

Proof. If $\operatorname{dim} H^{1}\left(A^{\bullet}, \omega\right)>0$, i.e. there exist linearly independent with $\omega$ form $\eta$ such that $\eta \wedge \omega=0$, then for any form $\omega^{\prime}$ in the space spanned by $\omega$ and $\eta$ one has $\operatorname{dim} H^{1}\left(A^{\bullet}, \omega^{\prime}\right)>0$. Let $\mathcal{V}$ be an irreducible component of $\mathcal{V}_{i}$ in (5.4.1) containing $\omega$ and having dimension $k \geq 2$. For the local system $L_{\omega^{\prime}}$ corresponding to each $\omega^{\prime} \in \mathcal{V}$ we have $\operatorname{dim} H^{1}\left(L_{\omega^{\prime}}\right)>0$. Indeed we can assume that $\omega^{\prime}$ is generic since this only decrease $H^{1}\left(L_{\omega^{\prime}}\right)$ (cf. [LY]). On the other hand for generic $\omega^{\prime}$, according to [ESV], we have $\operatorname{dim} H^{1}\left(A^{\bullet}, \omega^{\prime}\right)=\operatorname{dim} H^{1}\left(L_{\omega^{\prime}}\right)$. Therefore $L_{\omega}$ belongs to an irreducible component, say $V$, of the characteristic variety of $\mathbf{C}^{2}-\mathcal{L}$. Since the exponential map is a local homemorphism this component has the dimension equal to at least $k$. In fact the dimension of this component is exactly $k$. Assume to the contrary that this dimension is $l>k$ and let $f: \mathbf{C}^{2}-\mathcal{L} \rightarrow \mathbf{P}^{1}-\cup_{i=1}^{i=k+1} p_{i}$ be the map on a curve of general type (cf. (1.4.2) and [Ar], Prop. 1.7) corresponding to the component $V$. Then the pull back of form $H^{0}\left(\mathbf{P}^{1}, \Omega^{1}\left(\log \left(\cup p_{i}\right)\right)\right)$ gives $l$-dimensional space of forms on $\mathbf{C}^{2}-\mathcal{L}$ for which the wedge with $\omega$ is zero (note that the map $f^{*}$ is injective on $H^{1}$ ) and we have a contradiction. Let $t_{1}^{a_{1, j}} \ldots t_{r}^{a_{r, j}}=1(j=1, \ldots s)$ be the equations defining $V$ (cf. 4.2). Then $\omega$ belongs to the union of affine subspaces of $H^{0}\left(\mathbf{P}^{2}, \Omega^{1}\left(\log \left(\mathcal{L} \cup L_{\infty}\right)\right)\right.$ given by $Q_{j}=\Sigma a_{i, j} x_{i}=n_{j}, n_{j} \in \mathbf{Z}, j=1, \ldots, s$. Since $\operatorname{dim} H^{1}\left(A^{\bullet}, \lambda \omega\right)=$ $\operatorname{dim} H^{1}\left(A^{\bullet}, \omega\right), \lambda \in \mathbf{C}^{*}$ we see that $n_{j}=0$ for any $j$. Hence $\mathcal{V}$ is a linear space of dimension $k$ (and $i=\operatorname{dim} H^{1}\left(A^{\bullet}, \omega\right)=\operatorname{dim} H^{1}\left(L_{\omega}\right)=k-1$ ). 


\subsection{Combinatorial calculation of characteristic vari- eties.}

A consequence of the theorem 5.4 is that the irreducible components of the characteristic varieties containing the identity element of $H^{1}\left(\mathbf{C}^{2}-\mathcal{L}, \mathbf{C}^{*}\right)$ are determined by the cohomology of the complex (5.1.2). $H^{1}\left(A^{\bullet}, \omega\right)$ is the quotient of $\left\{\eta \in A^{1} \mid \eta \wedge \omega=0\right\}$ by the subspace spanned by $\omega$ and can be calculated as follows.

It is easy to see that a 2 -form is cohomologous to zero iff its integrals over all 2-cycles belonging to small balls about the multiple points of the arrangement are zeros. The group of such 2-cycles near a point which is the intersection of the lines $l_{i_{1}}, \ldots, l_{i_{m}}$ are generated by $\gamma_{i_{j}} \times\left(\gamma_{i_{1}}+\ldots .+\gamma_{i_{m}}\right)$. If $\left(\Sigma A_{i} \eta_{i}\right) \wedge \omega$ is cohomologous to zero in $\Omega^{2}\left(\mathbf{C}^{2}-\mathcal{L}\right)$ then vanishing of $\int A_{j} \eta_{j} \wedge s_{i} \eta_{i}$ over those 2 -cycles yields:

$$
A_{j}\left(\Sigma s_{i}\right)-\left(\Sigma A_{j}\right) s_{j}=0
$$

Therefore we obtain

$$
\begin{gathered}
A_{j}=C_{v} s_{j}\left(i f \Sigma_{v \in l_{j}} s_{j} \neq 0\right) \\
\Sigma A_{j}=0\left(i f \Sigma_{v \in l_{j}} s_{j}=0\right)
\end{gathered}
$$

for vertices $v$ of the arrangements. If we are looking for essential components of the characteristic variety (which we always can assume) then $s_{i} \neq 0$ and condition (5.5.2) can be replaced by

$$
\frac{A_{j}}{s_{j}}=\frac{A_{j^{\prime}}}{s_{j^{\prime}}} \quad \text { if } \quad \Sigma_{v \in l_{j}} s_{j} \neq 0
$$

Now for each subset of the set of vertices such that the system of equation (5.5.3) and supplementing it by equations (5.5.4) for vertices outside of selected subset has a solution non proportional to $\left(s_{1}, . ., s_{r}\right)$ we obtain a component $\mathcal{V}$ and hence corresponding component of the characteristic variety. We leave as an exercise to the reader to work out calculations of the characteristic varieties for the examples from section 3 using this method.

\section{References}


[1] [Ar] Arapura, D., (1997) Geometry of cohomology support loci for local systems, I, J. Alg. geom, 6, 563-597.

[2] [BE] Buchsbaum D. and Eisenbud D., (1977) What annihilates a module?, J.Algebra, 47, 231-243.

[3] [BHH] Barthel, G., Hirzebruch, F, Hofer T., (1987) Geradenkonfiguarationen und Algebraishe flächen, Vieweg Publishing, Wiesbaden.

[4] [BL] Blass P. and Lipman J., (1979) Remarks on adjoints and arithmetic genus of algebraic varieties, Amer. J. of Math, 101, 331-336.

[5] [Br] Brieskorn E., (1973) Sur les groups des tresses, In Sem. Bourbaki, Lect. Notes in Math. 317 Berlin, Heidelberg, New York, Springer, 21-44.

[6] [CE] Cartan H. and Eilenberg S., (1956) Homological Algebra, Princeton University Press, Princeton, N.J.

[7] [CS1] Cohen D. and Suciu A., (1995) On Milnor fibrations of arrangements, J.London Math. Soc, 51, 105-119.

[8] [CS2] Cohen D. and Suciu A., (1999) Characteristic varieties of arrangements. Math. Proc. Cambridge Phil. Soc. 127, 33-53.

[9] [De] Deligne P., (1971) Theorie de Hodge II, Publ. Math. I.H.E.S. 40, $5-58$.

[10] [ESV] Esnault H., Schechtman V., and Viehweg E., (1992) Cohomology of local systems on the complement to hyperplanes. Inv. math. 109, 557561.

[11] [F] Falk M., (1997) Arrangements and cohomology. Ann. Comb., 1, 135157.

[12] [GR] Grauert H. and Riemenschneider O., (1970) Inv. Math. 11, 263292.

[13] [Ha] Hartshorne R., (1977) Algebraic Geometry, Springer Verlag, Berlin Heidelberg New York. 
[14] [Hil] Hillman J., (1981) Alexander Ideals of Links Lecture Notes in Math. 895. Springer Verlag, Berlin Heidelberg New York.

[15] [Hi] Hironaka E., (1997) Multi-polynomial invariants of plane algebraic curves, Singularities and Complex Geometry, Studies in Advanced Mathematics, 5, AMS and International Press, 67-74.

[16] [I] Ishida M., (1983) The irregularity of Hirzebruch's examples of surfaces of general type with $c_{1}^{2}=3 c_{2}$. Math. Ann. 262, 407-420.

[17] [vK] van Kampen E.R., (1933) On the fundamental group of an algebraic curve, Amer. J. of Math, 55, 255-260.

[18] [Ko] Kollar J., (1995) Shafarevich maps and automorphic forms, Princeton University Press.

[19] [L1] Libgober A., (1982) Alexander polynomials of plane algebraic curves and cyclic multiple planes, Duke Math. J, 49. 833-851.

[20] [L2] Libgober A., (1983) Alexander invariants of plane algebraic curves, Proc. Symp. Pure Math. 40, AMS. Providence, RI, 135-143.

[21] [L3] Libgober A., (1992) On homology of finite abelian coverings, Topology and Applications, 43, 157-166.

[22] [L4] Libgober A., (1994) Groups which cannot be realized as fundamental groups of the complements to hypersurfaces in $\mathbf{C}^{N}$, Algebraic Geometry and Application, C.Bajaj editor, Springer Verlag, Berlin Heidelberg New York, 203-207.

[23] [L5] Libgober A., (1996) Position of singularities of hypersurfaces and the topology of their complements, J.Math. Sciences, 82, 3194-3210.

[24] [L6] Libgober A., (1999) Abelian Covers of projective plane, Singularity theory (Liverpool, 1996), xxi, London Math. Soc. Lecture Note Ser., 263, Cambridge Univ. Press, Cambridge, 281-289.

[25] [L7] Libgober A., (2001) Hodge decomposition of Alexander invariants. Preprint, 2001. 
[26] [LY] Libgober A., Yuzvinsky S., (2000) Cohomology of the BrieskornOrlik-Solomon algebra and local systems, Arrangements-Tokyo 1998, Adv. Stud. Pure Math., 27, Kinokuniya, Tokyo, 169-184.

[27] [LV] Loeser F.,and Vaquie M., (1990) Le polynome d'Alexander d'une courbe plane projective, Topology 29, 163-173.

[28] [N] Nori M., (1983) Zariski's conjecture and related problems. Ann. Sci. Ecole Norm. Sup. 16, 30 5-344.

[29] [Sab] Sabbah C., (1990) Module d'Alexander et $\mathcal{D}$-modules, Duke Math. Journal, 60, 729-814.

[30] [Sa] Sakuma M., (1995) Homology of abelian coverings of links and spatial graphs, Canadian Journal of Mathematics, 17, 201-224.

[31] [Se] Serre J.P., (1975) Algebre locale. Multiplicites, Lecture Notes in Mathemematics 11, (3rd edition), Springer Verlag, Berlin Heidelberg New York.

[32] [St] Steenrod N., (1945) Homology with local coefficients, Ann. Math., 44, 610-627.

[33] [SW] Sumners D. and Woods J., (1977) The monodromy of reducible plane curves, Inv. Math. 40, 107-141.

[34] [Z] Zariski O., (1971) Algebraic surfaces, Chapter.8, Springer Verlag, Berlin Heidelberg New York.

[35] [Zu] Zuo K., (1989) Kummer Oberlagerungen algebraischer Flächen. Bonner Mathematische Schriften, Bonn. 